\title{
Effective potential for SU(2) Polyakov loops and Wilson loop eigenvalues
}

\author{
Dominik Smith, ${ }^{a}$ Adrian Dumitru, ${ }^{b, c}$ Robert Pisarski, ${ }^{c, d}$ Lorenz von Smekal ${ }^{a, e}$ \\ ${ }^{a}$ Theoriezentrum, Institut für Kernphysik, TU Darmstadt, 64289 Darmstadt, Germany \\ ${ }^{b}$ Department of Natural Sciences, Baruch College, New York, NY 10010, USA \\ ${ }^{c}$ RIKEN/BNL Research Center, Brookhaven National Laboratory, Upton, NY 11973, USA \\ ${ }^{d}$ Department of Physics, Brookhaven National Laboratory, Upton, NY 11973, USA \\ ${ }^{e}$ Institut für Theoretische Physik, Justus-Liebig-Universität, 35392 Giessen, Germany
}

(Dated: August 21, 2018)

\begin{abstract}
We simulate $\mathrm{SU}(2)$ gauge theory at temperatures ranging from slightly below $T_{c}$ to roughly $2 T_{c}$ for two different values of the gauge coupling. Using a histogram method, we extract the effective potential for the Polyakov loop and for the phases of the eigenvalues of the thermal Wilson loop, in both the fundamental and adjoint representations. We show that the classical potential of the fundamental loop can be parametrized within a simple model which includes a Vandermonde potential and terms linear and quadratic in the Polyakov loop. We discuss how parametrizations for the other cases can be obtained from this model.
\end{abstract}

PACS numbers: 12.38.-t, 12.38.Gc, 12.38.Mh

\section{INTRODUCTION}

Understanding the deconfining phase transition of QCD is a long-standing problem in high energy physics. The situation is somewhat clearer in pure SU(N) YangMills theory, where it is understood that on length scales larger than $\sim 1 / T$ the relevant effective degrees of freedom are $\mathrm{SU}(\mathrm{N})$ spin variables (thermal Wilson loops) in three dimensions, obtained by compactifying the Euclidean time direction. In this picture, the deconfining phase transition manifests as spontaneous breaking of the $\mathrm{Z}(\mathrm{N})$ center symmetry [1-4].

Numerical simulations show that the interaction measure, $(e-3 p) / T^{4}$, times $T^{2} / T_{c}^{2}$, is approximately flat from $\sim 1.2 T_{c}$ to $\sim 4 T_{c}$, for up to six colors [12 15]. Similar behavior is observed in three space-time dimensions, for $(e-2 p) / T^{3}$ times $T / T_{c}$ [16, 17]. This shows that the leading corrections to an "ideal" gas term, $\sim T^{4}$ in four dimensions, and $\sim T^{3}$ in three dimensions, are terms $\sim T^{2}$, in both four and three dimensions. This differs from corrections due to a bag pressure as in the MIT bag model, which would be independent of temperature. This behavior can be reproduced within matrix models of the thermal Wilson loop [5 11] with coupling constants which are rather simple functions of $T$.

To understand this phenomenon better, it is useful to study the dynamics of the eigenvalues of the Wilson loops in different representations of the gauge group. The purpose of this work is to study the effective potential of bare Wilson loops and of their eigenvalues, in the fundamental and adjoint representations of SU(2), through ab-initio simulations in discretized space-time. Our goal is to shed some light on the validity of the perturbative ansatzes for the effective action, which are used in the construction of effective theories. Effective potentials for variables related to the phases of the eigenvalues of thermal Wilson loops have also been obtained from functional methods such as the Functional Renormalization Group or Dyson-Schwinger equations typically using background Landau-deWitt gauges [18, 19], and our results may be useful to benchmark these non-perturbative continuum quantum field-theoretic computations. Also, we wish to verify that the ansatz used to parametrize the results obtained in Refs. 20, 21] for the classical potential of a three dimensional matrix model of Wilson loops in the fundamental representation is valid also for the full gauge theory.

\section{SETUP}

We simulate pure $\mathrm{SU}(2)$ gauge theory using the standard Wilson action

$$
S=\beta \sum_{\square}\left(1-\frac{1}{2} \operatorname{Re} \operatorname{Tr} \mathbf{U}_{\square}\right)
$$

where the sum runs over all plaquettes of the four dimensional lattice. Lattice configurations are generated using an exact heatbath algorithm [22] which generates link variables according to the local Boltzmann distribution given by the staple matrix. $\mathrm{SU}(2)$ matrices are stored in the quaternionic representation

$$
U=a_{0} \mathbf{1}+i \sum_{j=1}^{3} a_{j} \sigma_{j} \quad, \sum_{j=0}^{3} a_{i}^{2}=1,
$$

where $a_{i} \in \mathbb{R}$ and $\sigma_{i}$ are the Pauli matrices. We construct thermal Wilson loops $\mathbf{L}(\mathbf{x})$ in the fundamental representation in the usual way by multiplying temporal links:

$$
\mathbf{L}(\mathbf{x})^{f}=\prod_{t=0}^{N_{t}-1} U_{4}(\mathbf{x}, t) .
$$

Loops in higher representations can be generated from the fundamental loop [23]. We currently only also consider the adjoint representation which is constructed as

$$
\mathbf{L}_{i j}^{a}=2 \operatorname{Tr}\left(T_{i} \mathbf{L}^{f} T_{j} \mathbf{L}^{\dagger f}\right),
$$


where $T_{i}$ are the three generators in the fundamental representation.

We are interested in the per-site effective potential $V_{\text {eff }}$ for the Polyakov loop $\ell \propto \operatorname{Tr} \mathbf{L}$ and for the eigenvalues of the Wilson line $\mathbf{L}$, in the fundamental or adjoint representations. In other words, $V_{\text {eff }}(\ell)$ describes fluctuations about the volume averaged mean field $\langle\ell\rangle$. The latter, in turn, determines the couplings $a(T), b(T), c(T)$ in $V_{\text {eff }}(\ell)$, see below.

In our work, "sites" are understood as the latticepoints of the three dimensional spin-system of the thermal Wilson loops. Our approach differs from the computation of a potential for volume averaged fields as in Refs. 24, 25].

We also comment on the difference of our approach to that of Ref. 26]. In this work they take the Wilson line to be constant in time. This is allowed, and in the continuum, corresponds to a static gauge, $\partial_{0} A_{0}=0$. There is still a residual gauge freedom to fix spatial gauge transformations at a given time. For example, one could fix to Coulomb gauge, $\partial_{i} A_{i}=0$. Instead, Ref. [26] computes an effective potential by assuming that the thermal Wilson line is constant in space. In the continuum this corresponds to setting $\partial_{i} A_{0}$ for all three spatial directions, and is not an allowable gauge condition. Because of this, their results are very different from ours or from Refs. [24, 25]. In particular, 26] finds a potential which is completely flat in the confined phase.

To obtain $V_{\text {eff }}$ we first compute the per-site probability distribution $P$ by histogramming each observable. From these distributions we obtain its classical or "constraint" effective potential $V_{0}=-\ln P$. The effective potential is then obtained from the Legendre transform of the moment-generating function $W(h)$ :

$$
W(h)=\ln \int d x \exp \left(-V_{0}(x)+h x\right) .
$$

Here, $x$ stands for the respective observable and the integral runs over the entire range of $x$. We obtain

$$
V_{\text {eff }}(\hat{x})=-\Gamma(\hat{x}),
$$

where

$$
\Gamma(\hat{x})=W(h(\hat{x}))-h(\hat{x}) \hat{x}, \quad \hat{x}=\frac{d W(h)}{d h} .
$$

In most cases considered here it is simpler to carry out the Legendre transformation via

$$
\Gamma(\hat{x})=\sup _{h}(\hat{x} h-W(h)) \text {. }
$$

This is our method of choice. ${ }^{1}$ We use Eq. (77) only in some special cases where analytical approximations to $W(h)$ can be easily obtained.

\footnotetext{
1 To be precise: in order to compute $\Gamma(\hat{x})$, for each data-set we take a proper parametrization of $V_{0}(x)$ as input. For a given $\hat{x}$
}

We use the string tension to fix the lattice spacing and thus the temperature $T=1 /\left(a N_{t}\right)$ in physical units. The exact procedure is discussed at length in appendix A (in all figures, the quoted $T / T_{c}$ are understood to have a relative uncertainty of less than $\sim 2 \%$ ). We change the temperature by varying $N_{t}$. The advantage of this fixed scale approach is that it allows us to clearly disentangle the effects of temperature changes and renormalization.

In the following we present a few formulae which shall be used throughout this work. Using Eqs. (21) and (4) with $T_{i}=\sigma_{i} / 2$ one can easily obtain the compact expression

$$
\mathbf{L}_{i j}^{a}=2\left(a_{i} a_{j}+a_{0} \sum_{k=1}^{3} \epsilon_{i j k} a_{k}+\delta_{i j}\left(a_{0}^{2}-\frac{1}{2}\right)\right),
$$

which relates the elements of the adjoint matrices to the quaternionic parameters $a_{i}$. From Eq. (9) it immediately follows that

$$
\operatorname{Tr} \mathbf{L}^{a}=\left|\operatorname{Tr} \mathbf{L}^{f}\right|^{2}-1 .
$$

From (2) it follows that the eigenvalues $\lambda_{1,2}$ of the fundamental loop are given by

$$
\lambda_{1,2}=a_{0} \pm \sqrt{a_{0}^{2}-1} .
$$

The eigenvalues form a pair of complex conjugates which lie on the unit circle. Also, they are related to the Polyakov loop $\ell$ by

$$
\ell^{f}=\frac{1}{2} \operatorname{Tr} \mathbf{L}^{f}=a_{0}=\frac{1}{2}\left(\lambda_{1}+\lambda_{2}\right) .
$$

Likewise, for the adjoint Wilson loop (which is a real 3by-3 matrix) one can easily show that the eigenvalues $\lambda_{1,2,3}$ are

$$
\lambda_{1}=1, \lambda_{2,3}=\frac{1}{2}\left(\operatorname{Tr} \mathbf{L}^{a}-1\right) \pm \sqrt{\frac{1}{4}\left(\operatorname{Tr} \mathbf{L}^{a}-1\right)^{2}-1} .
$$

The non-unit eigenvalues $\lambda_{2,3}$ again form a pair of complex conjugates with $|\lambda|=1$. Thus for both representations a single phase $\phi \sim \ln \lambda$ uniquely fixes all eigenvalues. The $\mathbf{L}$ are related by a similarity transform to $\operatorname{diag}\left(e^{i \phi}, e^{-i \phi}\right)$ and $\operatorname{diag}\left(1, e^{i \phi}, e^{-i \phi}\right)$ respectively, so that $\phi$ also fixes the trace of $\mathbf{L}$ through the relations

$$
\operatorname{Tr} \mathbf{L}^{f}=2 \cos \left(\phi^{f}\right), \quad \operatorname{Tr} \mathbf{L}^{a}=1+2 \cos \left(\phi^{a}\right) .
$$

we change $h$ from $h=-100$ to $h=100$ in steps of $d h=0.02$. For each $h, W(h)$ is computed from Eq. (5) using a $N$ point Gaussian quadrature with $N=2048$. We obtain the maximum $\hat{x} h-W(h)$ with respect to $h$. We repeat this process for 400 equidistant values of $\hat{x}$ in the domain of $\Gamma(\hat{x})$. We find that this procedure yields results which are stable under further increase of the resolution. 


\section{RESULTS}

We simulate $S U(2)$ gauge theory at $\beta=2.577856$ and $\beta=2.635365$, which corresponds to $T_{c}$ at $N_{t}=10,12$. The lattice spacing is $a \sqrt{\sigma}=0.140$ and $a \sqrt{\sigma}=0.116$, respectively. We use time-like lattice sizes of $N_{t}=$ $12,10,8,6$. For every lattice size and $\beta$ value several hundreds of independent ${ }^{2}$ lattice configurations were sampled from the equilibrium distribution. Bin sizes for the histograms were chosen such that the results appear as smooth lines at the resolutions of the figures presented here. All distributions $P$ presented here are normalized.

There is a subtle issue regarding ergodicity of simulations in the deconfined phase where the $Z(2)$ center symmetry is spontaneously broken: In principle, at any finite lattice volume, an ergodic algorithm which runs forever will tunnel infinitely often between the two groundstates. Hence, all probability densities $P$ will display a mixing of these groundstates and show no signs of a broken symmetry. The physics presented here however is concerned with the thermodynamic limit, for which the potential barrier between the groundstates becomes infinitely large. Tunneling events should therefore not occur, which strictly speaking violates ergodicity. From a practical point of view it is justified to ignore this problem as long as the lattice volumes considered are sufficiently large so that tunneling is strongly suppressed. One should take care however to ensure that this is truly the case for a given temperature, since such tunneling events occur more frequently as one approaches $T_{c}$ from above. One can achieve this, for instance, by monitoring the volume average of the Polyakov loop and looking for sign flips.

The results presented here are unaffected by this issue. The lowest temperatures in the deconfined phase which we consider here are well above $T_{c}$, so that even on a $32^{3} \times 4$ lattice tunneling is practically impossible and the infinite volume limit is well approximated. For each dataset discussed in this section simulations were done on lattices with $N_{x}=48$ and $N_{x}=64$. We find that both cases give identical results (indistinguishable histograms), which confirms that finite-volume effects play no role. Likewise, this confirms that statistical errors are negligible given the resolutions of the presented figures. Without restricting generality we chose to represent the symmetry broken phase as the state with $\langle\ell\rangle>0$.

\section{A. The fundamental representation}

We begin with the probability density $P(\ell)$ of the Polyakov loop $\ell=(1 / 2) \operatorname{Tr} \mathbf{L}$ in the fundamental representation. Fig. 1 shows $P(\ell)$ for $\beta=2.577856$ and $\beta=2.635365$ at $N_{t}=12,10,8,6$, which corresponds to

\footnotetext{
${ }^{2}$ Autocorrelations were investigated with the binning method [27].
}

temperatures ranging from slightly below $T_{c}$ to roughly $2 T_{c}$ (smaller $\beta$ corresponds to lower $T$ for fixed $N_{t}$ ). One

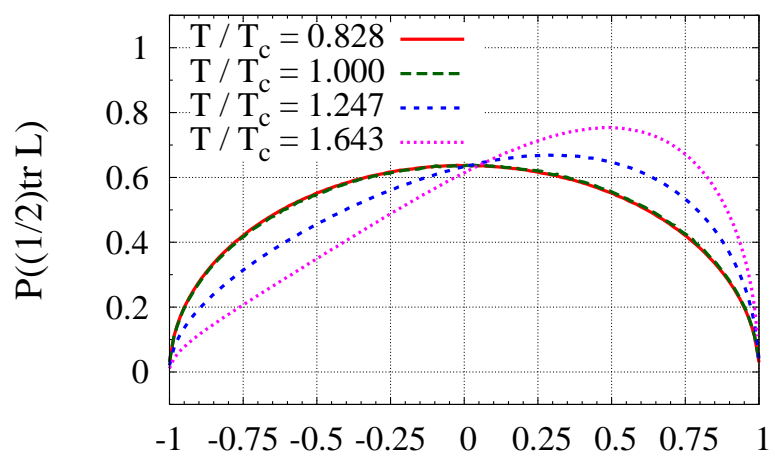

(1/2)tr L

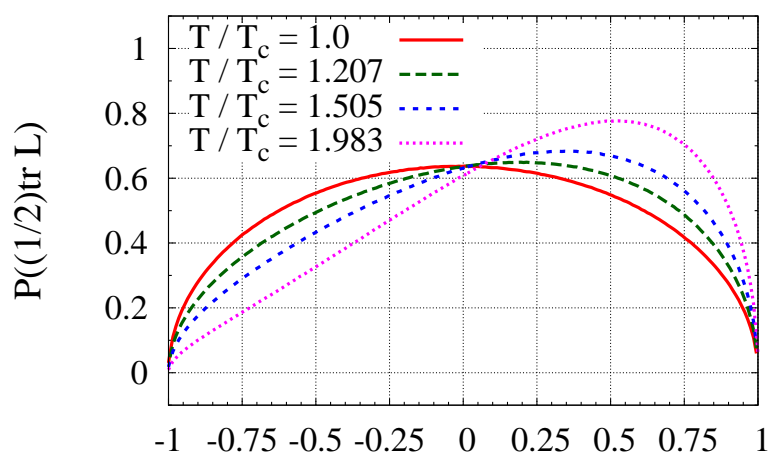

(1/2)tr L

FIG. 1: Distribution of the SU(2) Polyakov loop in the fundamental representation at $\beta=2.577856$ (top) and $\beta=$ 2.635365 (bottom). The different temperatures correspond to $N_{t}=12,10,8,6$ respectively.

can clearly see the effect of spontaneous breaking of the $Z(2)(\ell \rightarrow-\ell)$ symmetry: right at $T_{c}$ the distributions are symmetric around $\ell=0$. In fact, we have confirmed that they are identical for any $N_{t} \in[6, \ldots, 12]$ at the respective $\beta_{c}$. At higher temperature $P(\ell)$ is skewed and it develops a peak at non-zero $\ell$ which moves towards $\ell=1$ as one raises the temperature. In Fig. 2 one can see that the corresponding constraint effective potential $V_{0}(\ell)=-\ln P(\ell)$ gets tilted, which is exactly what one expects at a second order phase transition.

What is striking is that the lattice spacing affects the distribution. Comparing the simulations at the two different values of the coupling, $\beta=2.577856$ (corresponding to a larger lattice spacing) clearly approaches the perturbative vacuum $(\langle|\ell|\rangle \sim 1)$ much faster with increasing temperature. It is well known that the expectation value of the bare Polyakov loop is scale dependent and vanishes in the continuum limit (hence the need to construct renormalized loops [6, 23] ). However, here we show that 

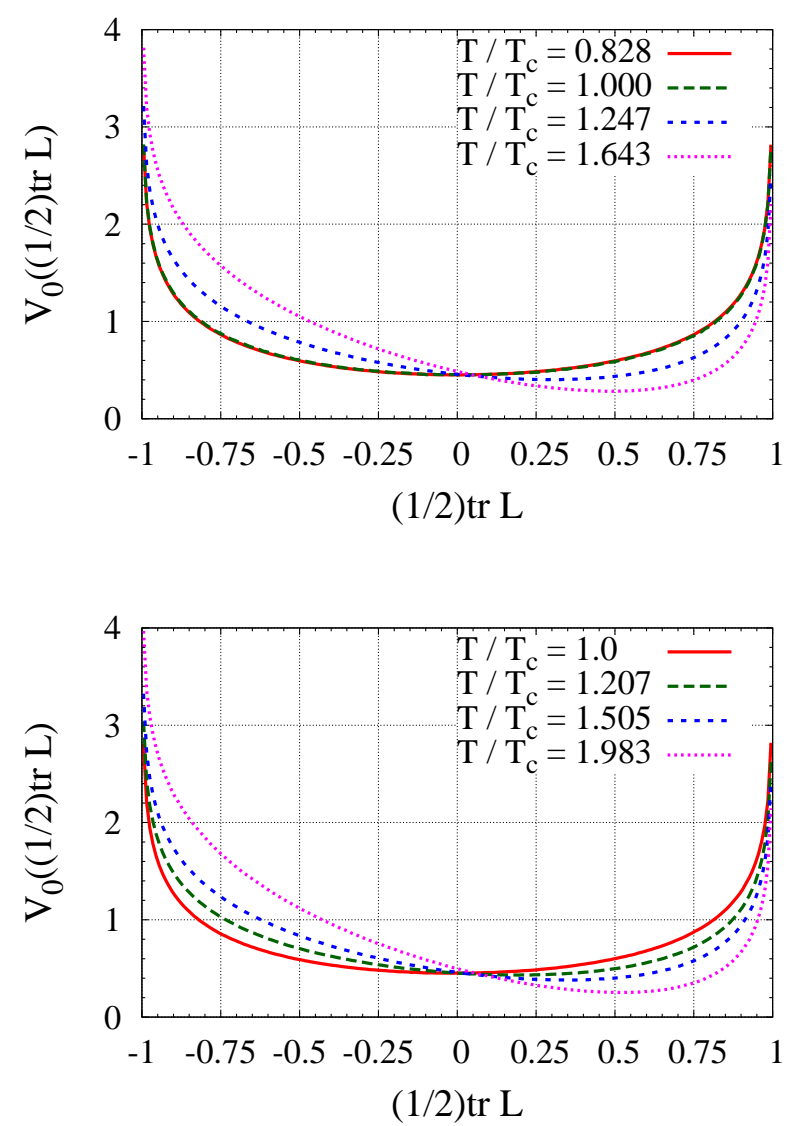

FIG. 2: Constraint effective potential of SU(2) Polyakov loop in fundamental representation at $\beta=2.577856$ (top) and $\beta=$ 2.635365 (bottom). The different temperatures correspond to $N_{t}=12,10,8,6$ respectively.

in fact the entire distribution is affected in a profound way. In the continuum $N_{t} \rightarrow \infty$ limit $P$ is deformed into the distribution at $T=T_{c}$. One way of thinking of this is that the $T=T_{c}$ distribution is an attractive ultraviolet fixed point of renormalization group transformations. What is also noteworthy is that the distribution appears not to change at $T<T_{c}{ }^{3}$

To make these statements quantitative we model the distributions using appropriate parametrizations. It turns out that the $T=T_{c}$ distribution is exactly

$$
P^{\left(T_{c}\right)}(\ell)=\frac{2}{\pi} \sqrt{1-\ell^{2}}
$$

which corresponds to a random walk on the $\mathrm{SU}(2)$ group manifold with the appropriate measure. The correspond-

\footnotetext{
${ }^{3}$ We do not rule out that there are small changes to the distribution below $T_{c}$ which we are not sensitive to. Such would be consistent with the findings of other authors [28, 29].
}

ing constraint potential is the Vandermonde potential [5]

$$
V_{0}^{\left(T_{c}\right)}(\ell)=-\frac{1}{2} \ln \left(1-\ell^{2}\right)-\ln \left(\frac{2}{\pi}\right) .
$$

At $T>T_{c}$, for the entire range of temperatures considered here, the following ansatz reproduces the simulated curves accurately:

$$
V_{0}(\ell)=V_{0}^{\left(T_{c}\right)}(\ell)+a(T)-b(T) \ell+c(T) \ell^{2} .
$$

This $V_{0}(\ell)$ corresponds to the potential for a single "local spin" $\ell(x)$ given that $\langle\ell\rangle \geq 0$. If instead one adopts the convention that $\langle\ell\rangle \leq 0$ by performing a $Z(2)$ transformation then $V_{0}(\ell)$ is obtained from the above by letting $b \rightarrow-b$.

The potential (17) corresponds to the distribution

$$
P(\ell)=\frac{2}{\pi} \sqrt{1-\ell^{2}} \exp \left(-a(T)+b(T) \ell-c(T) \ell^{2}\right) .
$$

We obtain the parameters $a, b, c$ from a $\chi^{2}$ fit. We do not plot the fits as they are indistinguishable from the simulation results. The temperature dependence of the parameters for both values of $\beta$ are shown in Fig. 3. It appears that the term linear in $\ell$ is dominant for the temperature range investigated here. It is also precisely this term which drives breaking of the $Z(2)$ symmetry (it in some sense describes the interaction of a particular site with the external field generated by the symmetry broken state of the lattice as a whole). The ansatz (17) is exactly of the form which was used in Refs. [20, 21] to parametrize the constraint effective potential for $\ell$ in a $3 D$ effective matrix theory of $S U(2)$ Wilson lines, with a similar behavior of the parameters $a, b, c$.

We obtain the effective potential $V_{\text {eff }}(\ell)$ by numerically solving Eq. (8), using the parametrization (17) of $V_{0}(\ell)$ as input. Obtaining the supremum numerically is straightforward. The integral in Eq. (5) is computed numerically via Gaussian quadrature. The results are shown in Fig.4. We have confirmed that the expectation value

$$
\langle\ell\rangle=\int_{-1}^{1} d \ell \ell P(\ell),
$$

coincides with the point at which $V_{\text {eff }}(\ell)$ is minimal, as it should.

From the same ensembles we obtain the per-site distribution of the phase $\phi$ of the eigenvalues of the Wilson loop L. Figs. 5 and 6 show the distributions $P(\phi)$ and the potentials $V_{0}(\phi)$ respectively. One can see that right at $T_{c}$ the distribution is peaked around $\mathbf{L} \sim \operatorname{diag}(i,-i)$, which is the state of maximal eigenvalue repulsion. Increasing the temperature drives the peak towards $\mathbf{L} \sim \operatorname{diag}(1,1)$ though eigenvalue repulsion persists even above $T_{c}$. Such an effect is known to occur also in matrix models of the Wilson line [20, 30, 31].

We can transform the parametrization of $P(\ell)$ given in Eq. (18) to $P(\phi)$. Eq. (14) provides a one-to-one mapping 

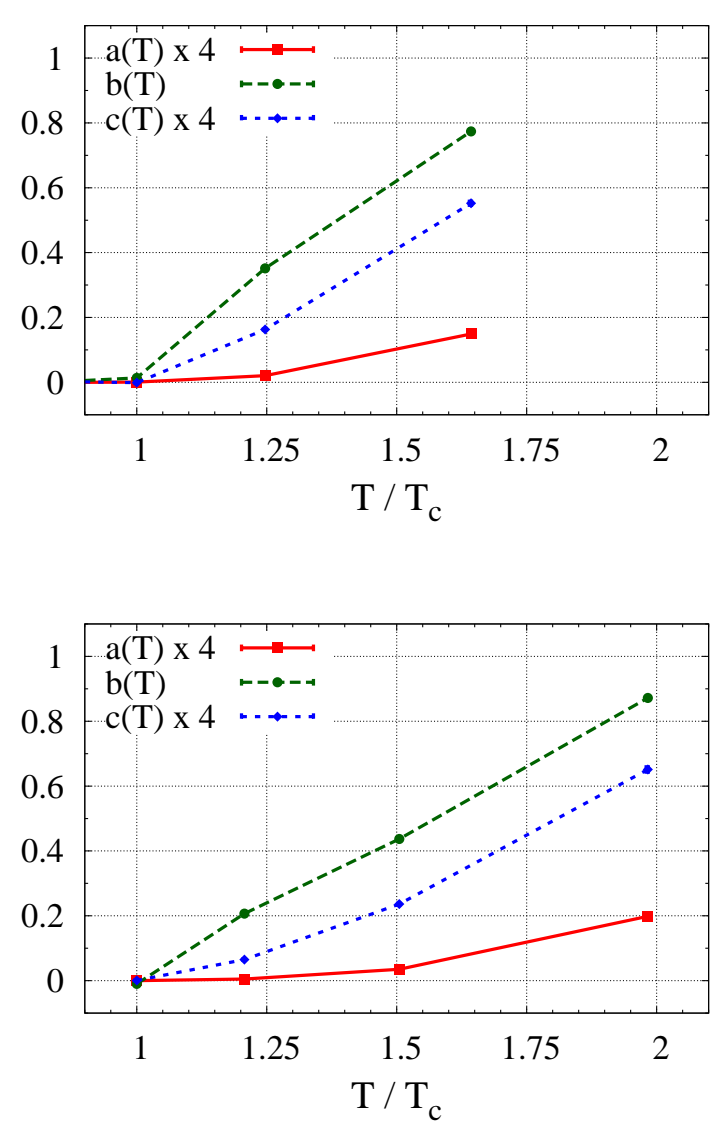

FIG. 3: Fit coefficients for $\beta=2.577856$ (top) and $\beta=$ 2.635365 (bottom). $a(T)$ and $c(T)$ are scaled up by factors of 4. The connecting lines are there to guide the eye only but do not represent actual models.

from $\ell=[-1, \ldots, 1]$ to $\phi=[0, \ldots, \pi]$; we can thus use the standard integral transformation law to obtain $P(\phi)$ from our models (15) and (18). $\phi=[-\pi, \ldots, 0]$ maps into the same distribution $P(\ell)$, thus the restriction to positive $\phi$ is legitimate. Using

$$
\int_{-1}^{1} d \ell P(\ell)=\int_{\phi(\ell=-1)}^{\phi(\ell=1)} P(\ell(\phi)) \frac{d \ell}{d \phi} d \phi=\int_{\pi}^{0} P(\phi) d \phi,
$$

we arrive at

$$
P^{\left(T_{c}\right)}(\phi)=\frac{2}{\pi} \sin ^{2} \phi=\frac{1}{\pi}(1+\cos (2 \phi+\pi)),
$$

for the distribution right at $T_{c}$ and

$$
\begin{aligned}
P(\phi)= & \frac{2}{\pi} \sin ^{2} \phi \\
& \times \exp \left(-a(T)+b(T) \cos (\phi)-c(T) \cos ^{2}(\phi)\right),
\end{aligned}
$$

for the general case. The parameters $a, b, c$ are unchanged. Eqs. (21) and (22) again describe the simulated
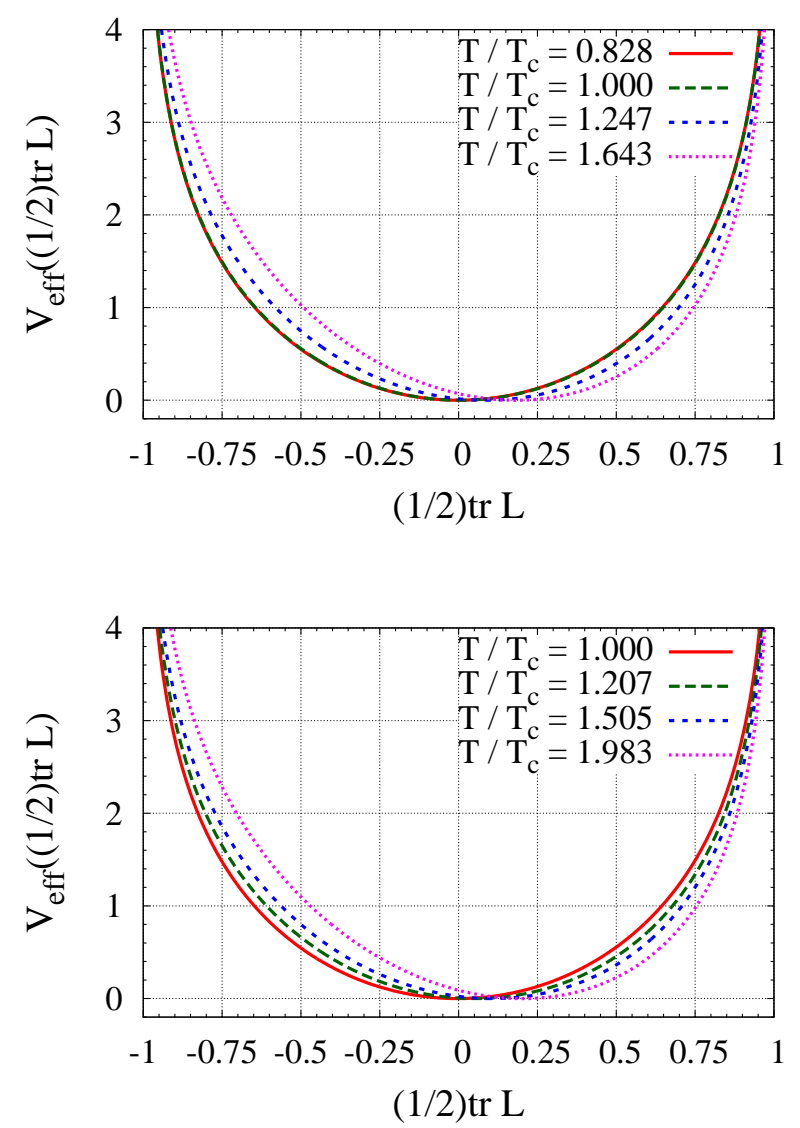

FIG. 4: Effective potential for the SU(2) Polyakov loop in the fundamental representation at $\beta=2.577856$ (top) and $\beta=2.635365$ (bottom). The quoted temperatures correspond to $N_{t}=12,10,8,6$ respectively.

results to such accuracy that the lines are indistinguishable and thus the fit-curves are left out of the figures. We again use our model function

$$
\begin{aligned}
& V_{0}(\phi)= \\
& -\ln \left(\frac{2}{\pi} \sin ^{2} \phi\right)+a(T)-b(T) \cos (\phi)+c(T) \cos ^{2}(\phi),
\end{aligned}
$$

to obtain $V_{\text {eff }}(\phi)$. The Legendre transformation (8) is carried out numerically, as before. The results are shown in Fig. 7.

In order to make our results portable (to compare them with other calculations or use them as input for effective theories) we would like to obtain analytical expressions for $V_{\text {eff }}(\phi)$ and $V_{\text {eff }}(\ell)$. One possible approach is to again use polynomial models (together with the Vandermonde contribution) and adjust the parameters to fit the data. It turns out, however, that this approach is not satisfactory as it requires high-order polynomials and many parameters in both cases. Given how well our models (18) and (22) described the simulated results for $P(\ell)$ and $P(\phi)$, it would therefore be nice if the Legendre transfor- 

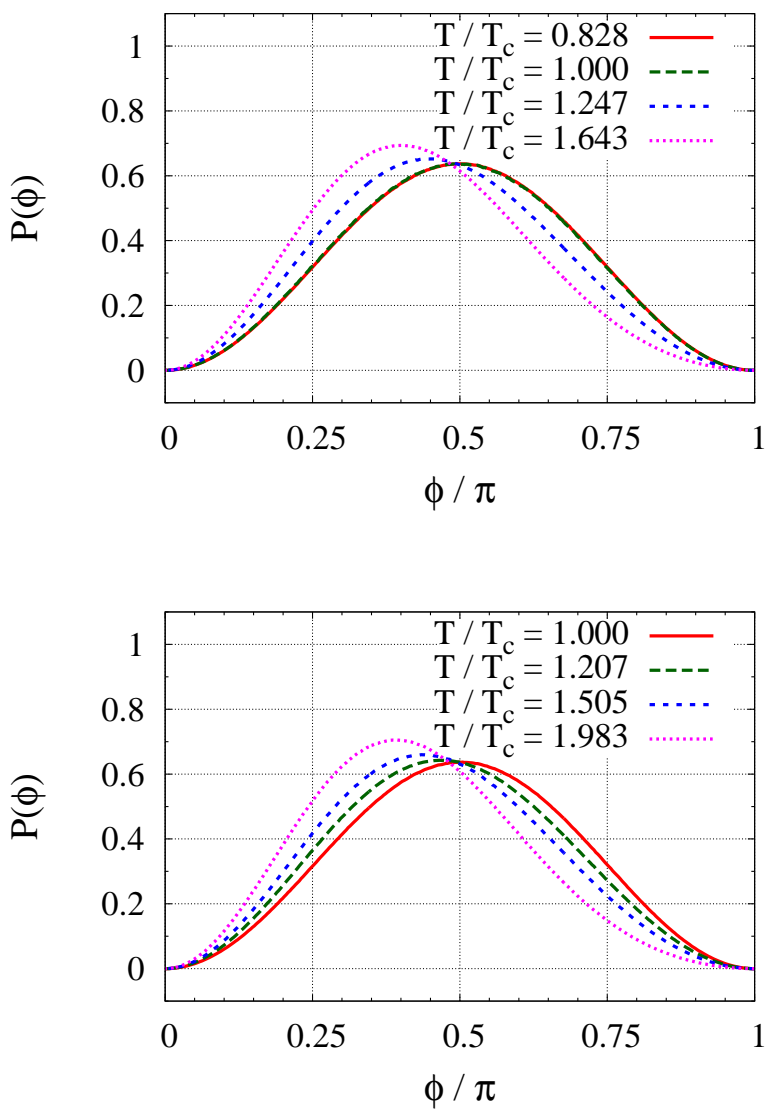

FIG. 5: Distribution of the phase of SU(2) Wilson loop eigenvalues in the fundamental representation at $\beta=2.577856$ (top) and $\beta=2.635365$ (bottom). The quoted temperatures correspond to $N_{t}=12,10,8,6$ respectively.

mation (77) could be carried out exactly. Unfortunately this is not possible in closed form. Nevertheless, an approximate solution can still be obtained using the saddle point approximation. The next-to-leading order result is easily obtained, and we will compare this result to our data in the following.

Consider a stationary point $x_{0}$ of the exponential appearing in Eq. (5) such that

$$
V_{0}^{\prime}\left(x_{0}\right)=h .
$$

We can expand the exponent in Eq. (5) around this point. Neglecting terms containing derivatives of higher than second order we can carry out the integral:

$$
\begin{aligned}
& W(h)=\ln \int_{-\infty}^{\infty} d x \exp \left(-V_{0}(x)+h x\right) \\
& \approx \ln \int_{-\infty}^{\infty} d x \exp \left(-V_{0}\left(x_{0}\right)+h x_{0}-\frac{1}{2} V_{0}^{\prime \prime}\left(x_{0}\right)\left(x_{0}-x\right)^{2}\right) \\
& \quad=-V_{0}\left(x_{0}\right)+h x_{0}+\frac{1}{2} \ln \left(\frac{V_{0}^{\prime \prime}\left(x_{0}\right)}{2 \pi}\right)
\end{aligned}
$$

and

$V_{0}^{\prime \prime}(\phi)=\frac{2}{\sin ^{2}(\phi)}+b(T) \cos (\phi)+2 c(T)\left(\sin ^{2}(\phi)-\cos ^{2}(\phi)\right)$.

In Figs. 8 and 9 we compare the NLO saddle point approximation to the exact results for both $V_{\text {eff }}(\ell)$ and 

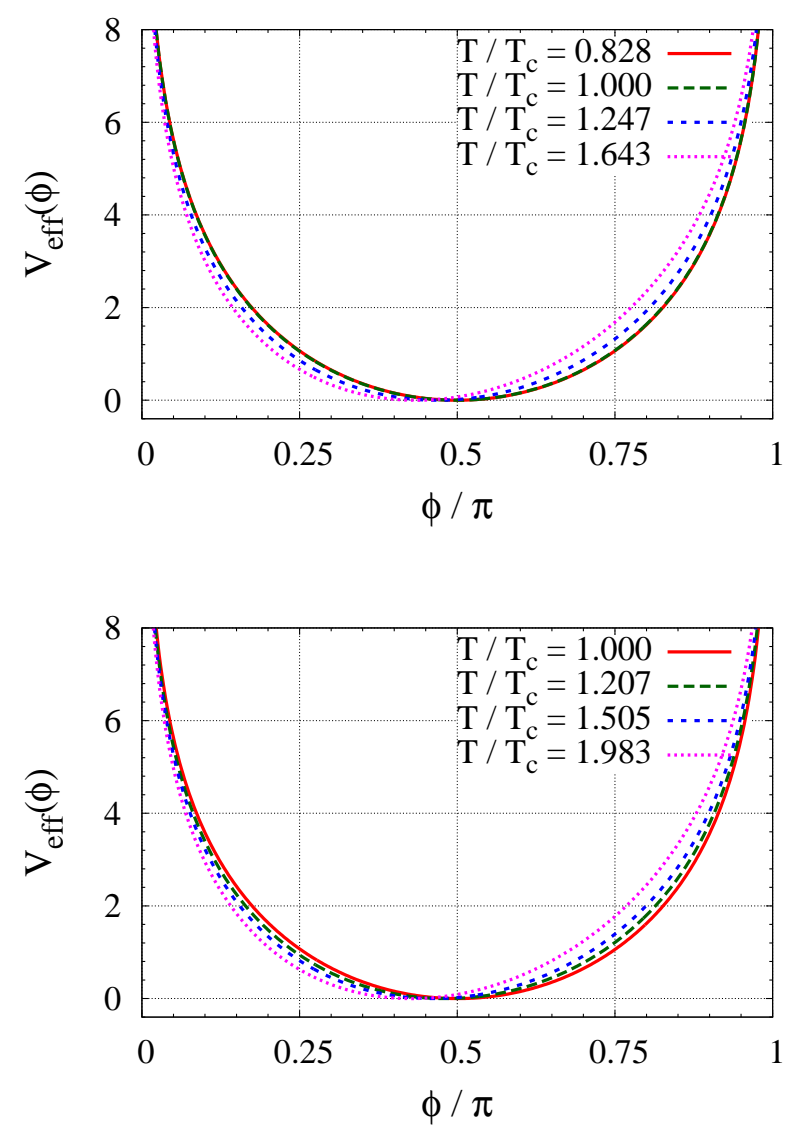

FIG. 7: Effective potential of phase of $\mathrm{SU}(2)$ Wilson loop eigenvalues in fundamental representation at $\beta=2.577856$ (top) and $\beta=2.635365$ (bottom). The different temperatures correspond to $N_{t}=12,10,8,6$ respectively.

$V_{\text {eff }}(\phi)$. The approximate curves are corrected by an additive shift to account for the loss of normalization of $P$ which occurs. The shift is such that $V_{\text {eff }}$ is moved to zero at its minimum. In both cases $N_{t}=6$ is chosen as an example. It appears that the approximation is quite good.

Lastly, we investigate how well the classical potentials $V_{0}(\ell)$ and $V_{0}(\phi)$ can be approximated by Taylor polynomials around the confined $(\phi= \pm \pi / 2)$ or deconfined $(\phi=0, \pi)$ vacua. Expanding about $\phi=\pi / 2$ we obtain

$$
\begin{aligned}
V_{0,1}(\phi) \approx & -\ln \left(\frac{2}{\pi}\right)+a(T)+b(T)\left(\phi-\frac{\pi}{2}\right) \\
& +(c(T)+1)\left(\phi-\frac{\pi}{2}\right)^{2}-\frac{b(T)}{6}\left(\phi-\frac{\pi}{2}\right)^{3} \\
& +\left(\frac{1}{6}-\frac{c(T)}{3}\right)\left(\phi-\frac{\pi}{2}\right)^{4} .
\end{aligned}
$$

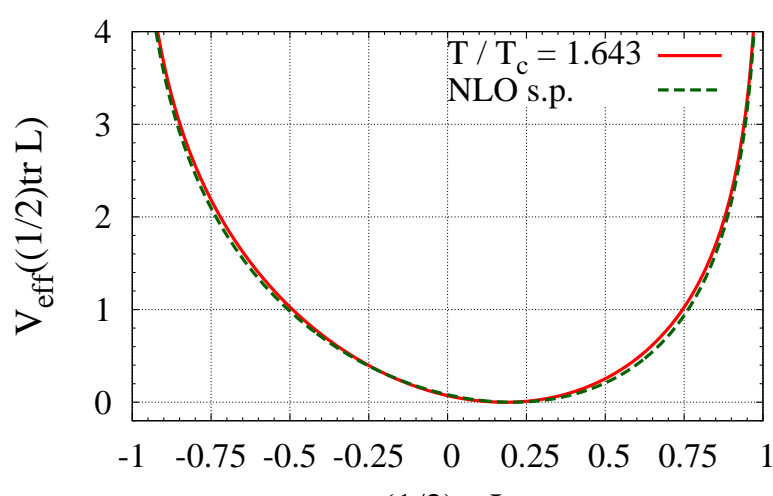

$(1 / 2) \operatorname{tr~L}$

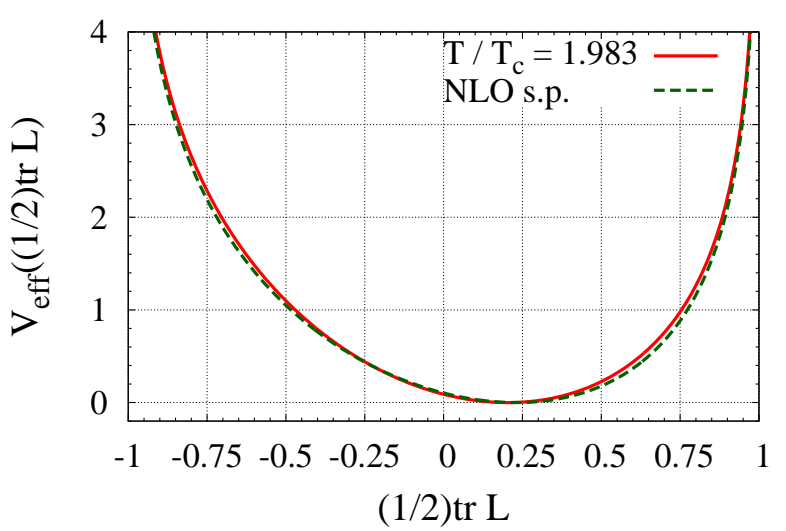

FIG. 8: Effective potential for $\mathrm{SU}(2)$ Polyakov loop in fundamental representation at $\beta=2.577856$ (top) and $\beta=$ 2.635365 (bottom). $N_{t}=6$ simulation result compared to NLO saddle point expansion. The SP curve has been shifted vertically to bring the minimum to zero.

Likewise, about $\phi=0$

$$
\begin{aligned}
V_{0,2}(\phi) \approx & -\ln \left(\frac{2}{\pi} \sin ^{2}(\phi)\right)+a(T)-b(T)+c(T) \\
& +\left(\frac{b(T)}{2}-c(T)\right) \phi^{2} \\
& +\left(\frac{c(T)}{3}-\frac{b(T)}{24}\right) \phi^{4}
\end{aligned}
$$

The logarithmic term has not been expanded since it diverges at $\phi=0$.

Finally, an expansion around $\phi=\pi$ yields

$$
\begin{aligned}
V_{0,3}(\phi) \approx & -\ln \left(\frac{2}{\pi} \sin ^{2}(\phi)\right)+a(T)+b(T)+c(T) \\
& +\left(\frac{-b(T)}{2}-c(T)\right)(\phi-\pi)^{2} \\
& +\left(\frac{c(T)}{3}-\frac{-b(T)}{24}\right)(\phi-\pi)^{4}
\end{aligned}
$$



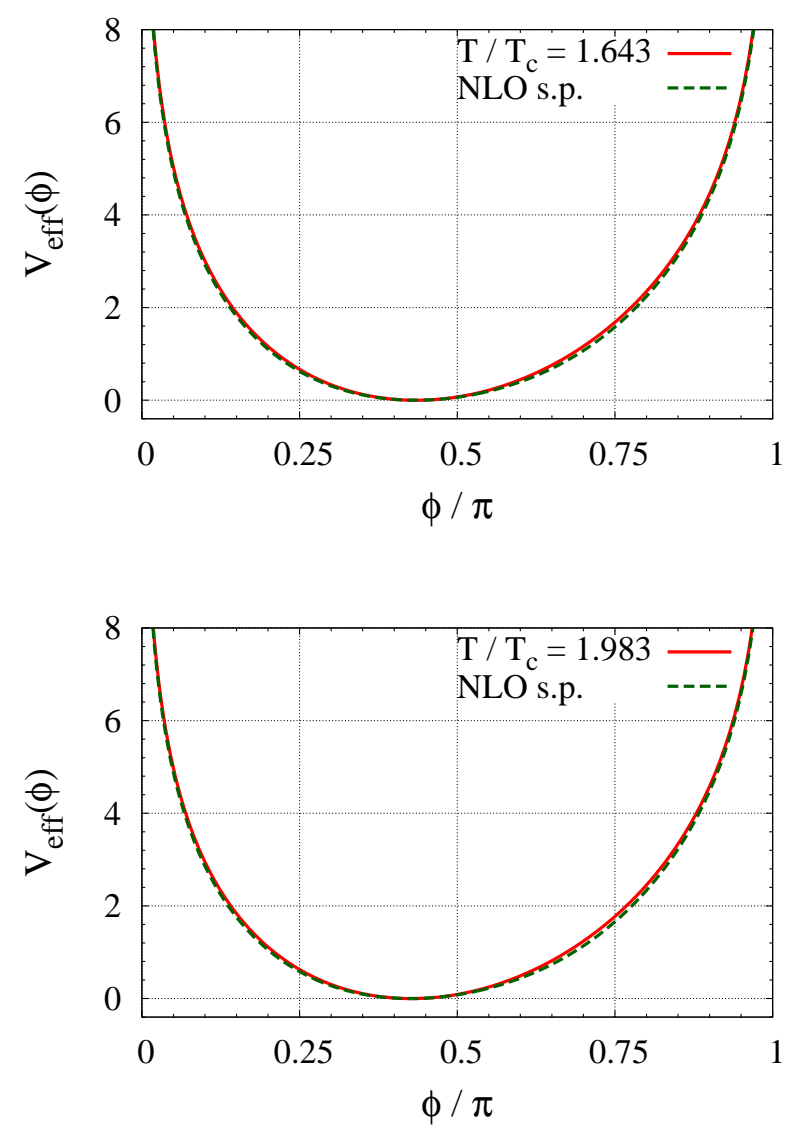

FIG. 9: Effective potential for the phase of $\mathrm{SU}(2)$ Wilson loop eigenvalues in the fundamental representation at $\beta=$ 2.577856 (top) and $\beta=2.635365$ (bottom). $N_{t}=6$ result compared to NLO saddle point expansion. The SP curve has been shifted vertically to bring the minimum to zero.

Note that (32) is nothing but the $\mathrm{Z}(2)$ transform of (31) corresponding to $\phi \rightarrow \pi-\phi$ and $b \rightarrow-b$.

Fig. 10 compares these expansions to the simulation results for $N_{t}=6$, which corresponds to the highest temperature for each respective $\beta$. It can be seen that Eq. (30) works well, even if one terminates the expansion at $\mathcal{O}(2)$. The approximation smoothly approaches the simulation results when at higher orders, and $\mathcal{O}(4)$ reproduces the exact result to high accuracy. Convergence is slower for Eq. (31) and very slow for (32). The latter is not extremely suprising since $\phi=\pi$ is the wrong groundstate. A similar expansion can be constructed for the logarithmic contribution to $V_{0}(\ell)$. Expanding Eq. (17) to $\mathcal{O}(6)$ about $\ell=0$ we get

$$
\begin{aligned}
V_{0,1}(\ell) \approx & -\ln \left(\frac{\pi}{2}\right)+a(T)-b(T) \ell \\
& +\left(c(T)+\frac{1}{2}\right) \ell^{2}+\frac{1}{4} \ell^{4}+\frac{1}{6} \ell^{6} .
\end{aligned}
$$

Fig. 11] shows the convergence of this expansion towards
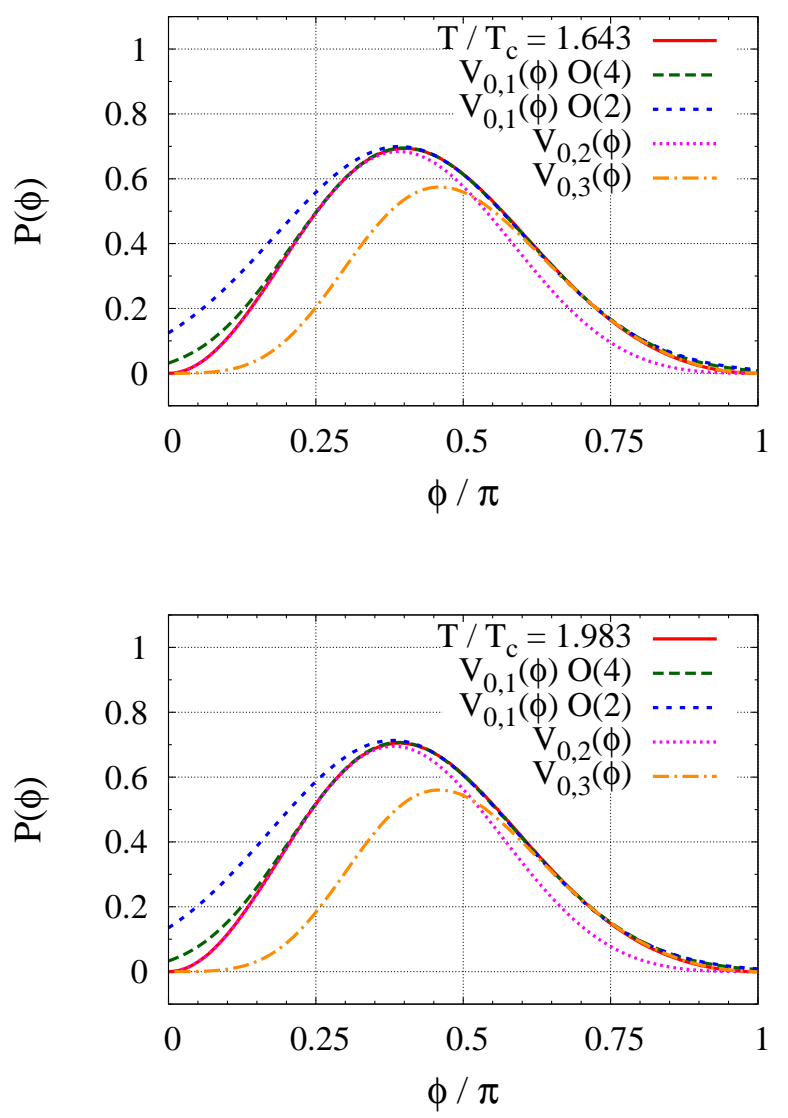

FIG. 10: Distribution of phase of SU(2) Wilson loop eigenvalues in the fundamental representation at $\beta=2.577856$ (top) and $\beta=2.635365$ (bottom). $N_{t}=6$ results compared to Taylor expansions.

the simulated curves for $N_{t}=6$. High orders are required to accurately reproduce the data.

\section{B. The adjoint representation}

From the same ensembles, we can also construct distributions $P\left(\operatorname{tr} \mathbf{L}^{a}\right)$ (here without any normalization factors before the trace) for the adjoint loop as well as for its eigenvalues. The results are shown in Figs. 12 and 14. respectively. Figs. 13 and 15 show the corresponding potentials $V_{0}=-\ln P$. What is striking is that temperature effects appear to be small. Nevertheless, a slight suppression of $P(\phi)$ around $\phi=\pi$ can be observed with increasing temperature.

We parametrize these results in a way similar to those for the fundamental representation. We begin by transforming the parametrization of $P(\phi)$ given in Eq. (22) to the adjoint representation. Once this is achieved, $P(\operatorname{tr} \mathbf{L})$ can be easily obtained. To approach this problem we require a one-to-one mapping $\phi^{f} \mapsto \phi^{a}$ from the fundamen- 


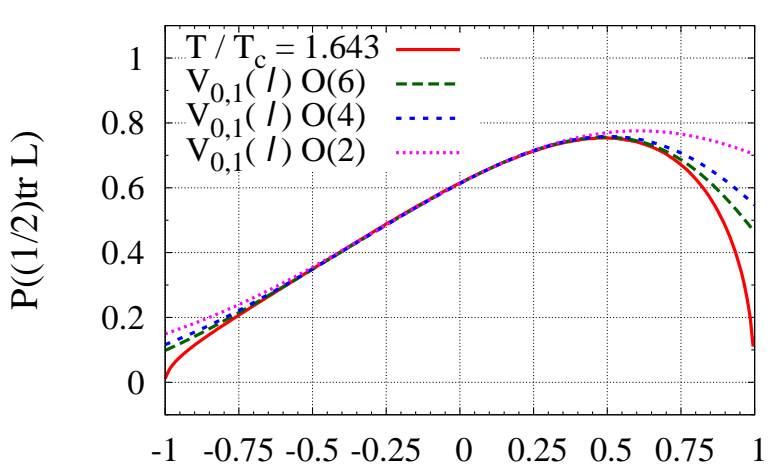

$(1 / 2) \operatorname{tr} \mathrm{L}$

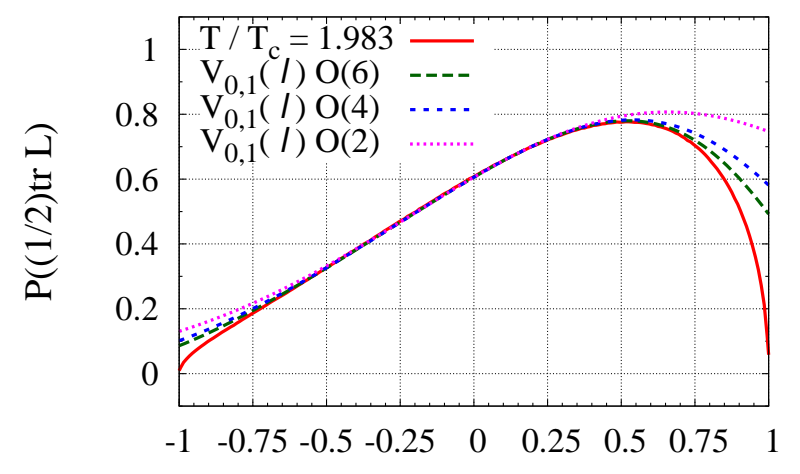

$(1 / 2) \operatorname{tr} \mathrm{L}$

FIG. 11: Distribution of SU(2) Polyakov loop in the fundamental representation at $\beta=2.577856$ (top) and $\beta=$ 2.635365 (bottom). $N_{t}=6$ results compared to Taylor expansions.

tal to the adjoint phase. To construct such a mapping a few subtleties arise. Eq. (10) implies that

$$
\cos ^{2}\left(\phi^{f}\right)=\frac{1}{2}\left(1+\cos \left(\phi^{a}\right)\right)
$$

Completing one full rotation in the fundamental phase $\phi^{f}$ (from $\phi=-\pi$ to $\phi=+\pi$ ) generates two full cycles of the square cosine on the left-hand side of Eq. (34), whereas one rotation of the adjoint phase $\phi^{a}$ is only one cycle of the right-hand side. Both sides are symmetric around $\phi=0$, thus it is sufficient to consider only $\phi^{f} \in$ $[0, \pi]$ which can be mapped to one full rotation of the adjoint phase. Second, the left-hand side possesses an inflection point at $\phi^{f}=\frac{\pi}{2}$. Thus the mapping must be constructed piece-wise in the intervals $\phi \in\left[0, \frac{\pi}{2}\right]$ and $\phi \in$ $\left[\frac{\pi}{2}, 0\right]$. Taking positive roots in Eq. (34) and considering that

$$
+\sqrt{\frac{1}{2}(1+\cos (x))}= \begin{cases}\cos \left(\frac{x}{2}\right) & : x \in[0, \pi] \\ -\cos \left(\frac{x}{2}\right) & : x \in[\pi, 2 \pi]\end{cases}
$$
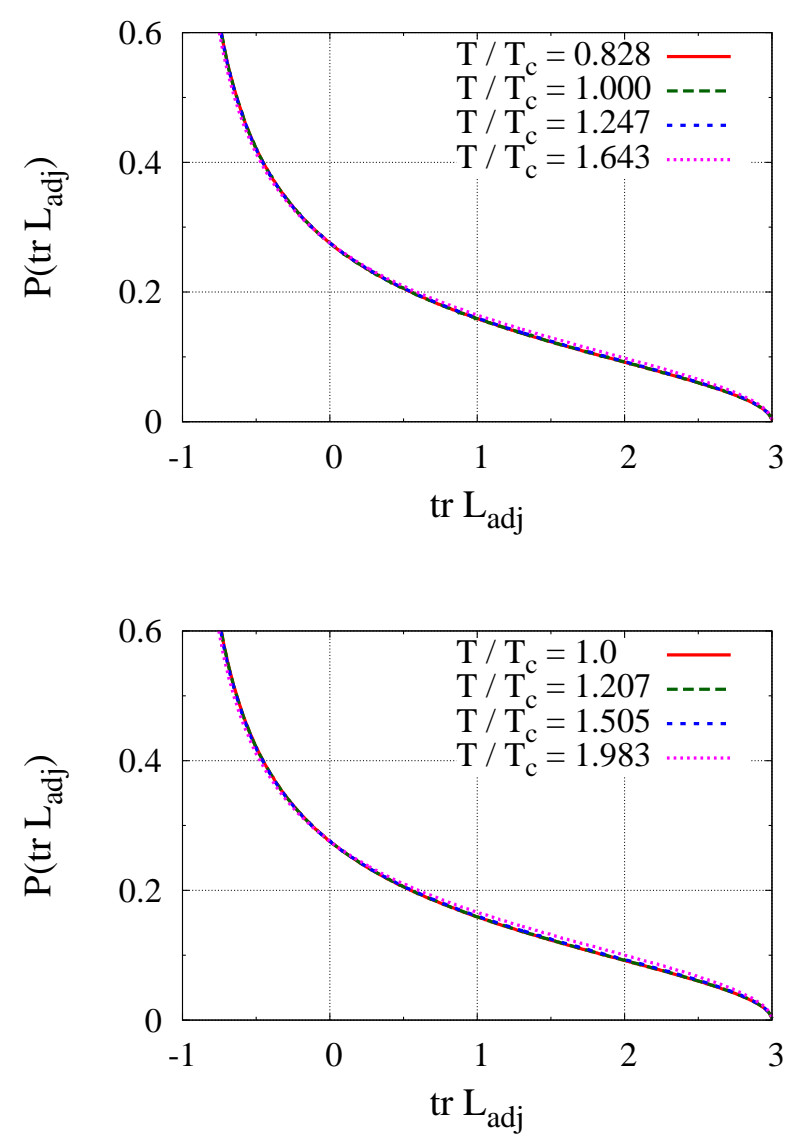

FIG. 12: Distribution of SU(2) Polyakov loop in adjoint representation at $\beta=2.577856$ (top) and $\beta=2.635365$ (bottom). The different temperatures correspond to $N_{t}=12,10,8,6$ respectively.

we obtain

$$
\cos \left(\phi^{f}\right)= \begin{cases}\cos \left(\frac{\phi^{a}}{2}\right) & : \phi^{f} \in[0, \ldots, \pi / 2] \\ -\cos \left(\frac{\phi^{a}}{2}\right) & : \phi^{f} \in[\pi / 2, \ldots, \pi]\end{cases}
$$

and thus

$$
\phi^{f}= \begin{cases}\frac{\phi^{a}}{2} & : \phi^{f} \in[0, \ldots, \pi / 2] \\ \pi-\frac{\phi^{a}}{2} & : \phi^{f} \in[\pi / 2, \ldots, \pi]\end{cases}
$$

This is a one-to-one mapping, covering the entire range of both $\operatorname{tr} \mathbf{L}^{f}$ and $\operatorname{tr} \mathbf{L}^{a}$ and which bijectively maps $\operatorname{tr} \mathbf{L}^{f} \mapsto$ $\operatorname{tr} \mathbf{L}^{a}$.

We can now carry out the transformation according to

$$
\begin{aligned}
\int_{0}^{\pi} d \phi^{f} P\left(\phi^{f}\right) & =\int_{0}^{\frac{\pi}{2}} d \phi^{f} P\left(\phi^{f}\right)+\int_{\frac{\pi}{2}}^{\pi} d \phi^{f} P\left(\phi^{f}\right) \\
& =\int_{0}^{\pi} P\left(\phi^{a}\right) d \phi^{a}
\end{aligned}
$$



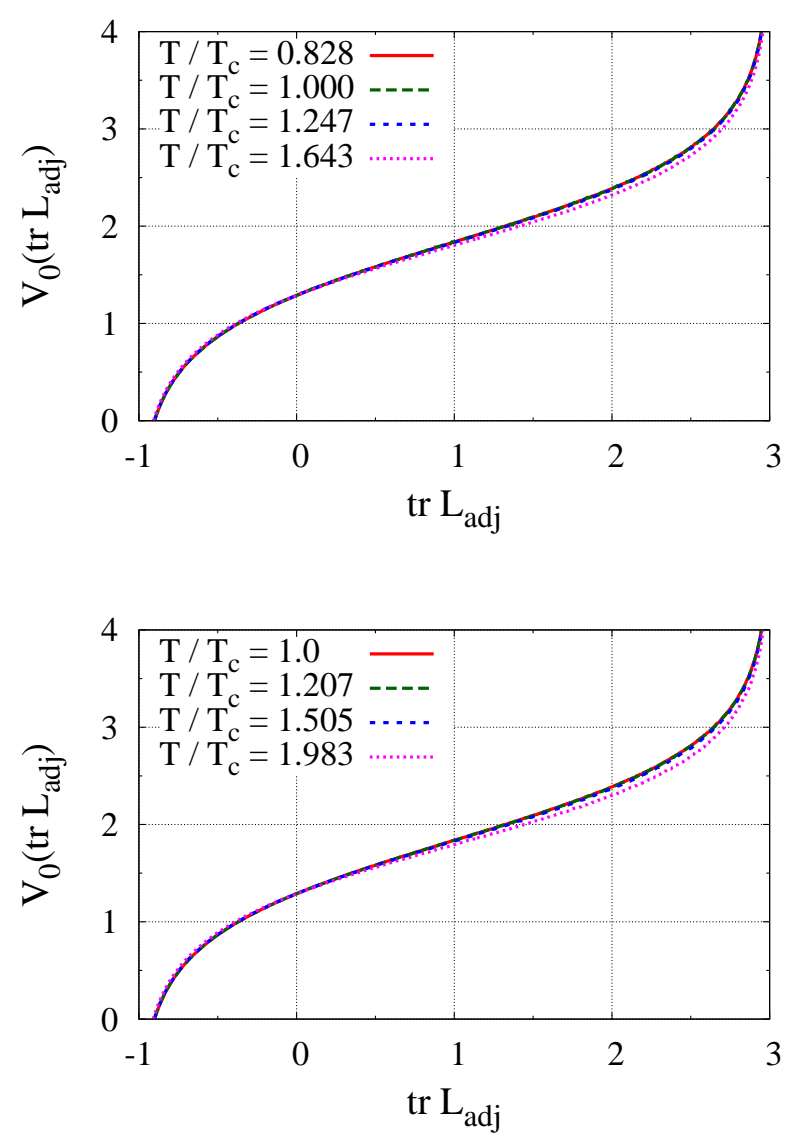

FIG. 13: Constraint effective potential of SU(2) Polyakov loop in adjoint representation at $\beta=2.577856$ (top) and $\beta=2.635365$ (bottom). The different temperatures correspond to $N_{t}=12,10,8,6$ respectively.

We obtain for the adjoint phase $\phi^{a}$

$$
\begin{aligned}
P\left(\phi^{a}\right)= & P^{\left(T_{c}\right)}\left(\phi^{a}\right) \exp \left(-a(T)-c(T) \cos ^{2}\left(\frac{\phi^{a}}{2}\right)\right) \\
& \times \cosh \left(b(T) \cos \left(\frac{\phi^{a}}{2}\right)\right),
\end{aligned}
$$

with

$$
P^{\left(T_{c}\right)}\left(\phi^{a}\right)=\frac{1}{\pi} \sin \left(\phi^{a}\right) \sqrt{\frac{1-\cos \left(\phi^{a}\right)}{1+\cos \left(\phi^{a}\right)}}=\frac{2}{\pi} \sin ^{2}\left(\frac{\phi^{a}}{2}\right) .
$$

Likewise, for the adjoint loop $\operatorname{tr} \mathbf{L}^{a}$ we obtain

$$
\begin{aligned}
P\left(\operatorname{tr} \mathbf{L}^{a}\right)= & P^{\left(T_{c}\right)}\left(\operatorname{tr} \mathbf{L}^{a}\right) \exp \left(-a(T)-\frac{c(T)}{4}\left(1+\operatorname{tr} \mathbf{L}^{a}\right)\right) \\
& \times \cosh \left(\frac{b(T)}{2} \sqrt{1+\operatorname{tr} \mathbf{L}^{a}}\right),
\end{aligned}
$$

with

$$
P^{\left(T_{c}\right)}\left(\operatorname{tr} \mathbf{L}^{a}\right)=\frac{1}{2 \pi} \sqrt{\frac{3-\operatorname{tr} \mathbf{L}^{a}}{1+\operatorname{tr} \mathbf{L}^{a}}}
$$
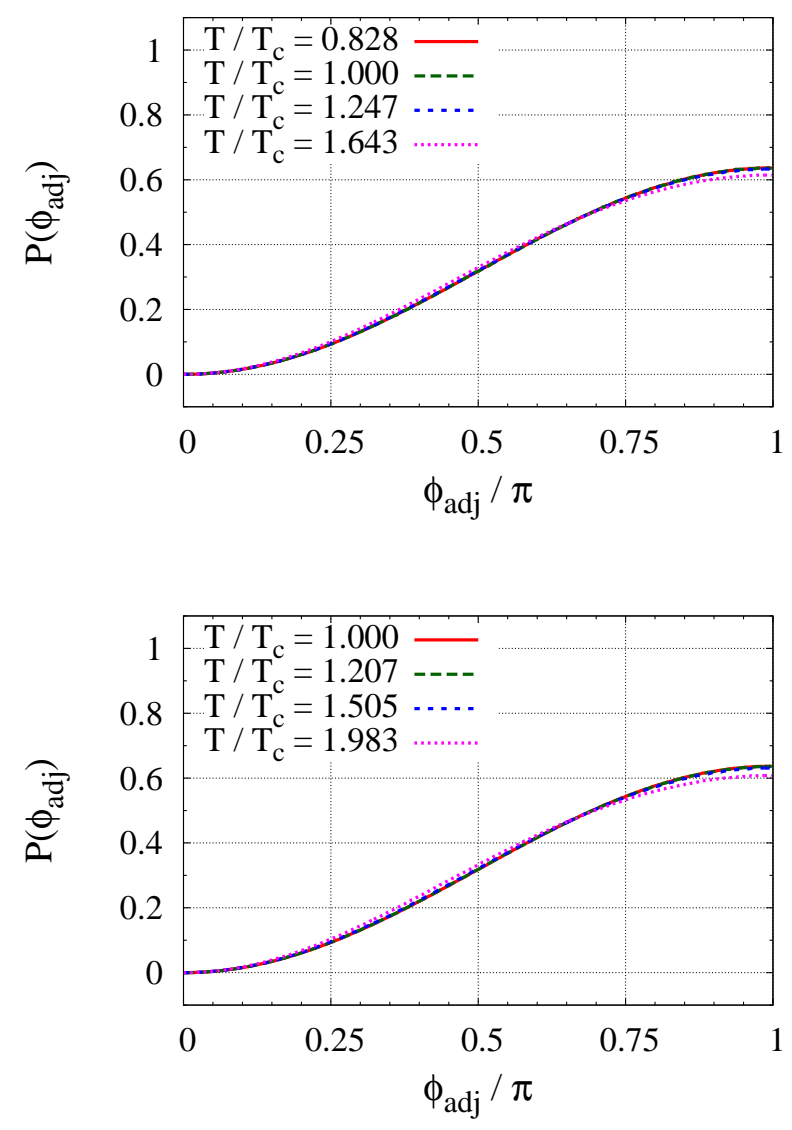

FIG. 14: Distribution of phase of SU(2) Wilson loop eigenvalues in adjoint representation at $\beta=2.577856$ (top) and $\beta=2.635365$ (bottom). The different temperatures correspond to $N_{t}=12,10,8,6$ respectively.

The simulation results (Figs. 12 and 14) are again reproduced to such accuracy that the curves are virtually indistinguishable. We carry out the Legendre transformations (8) to obtain $V_{\text {eff }}\left(\operatorname{tr} \mathbf{L}^{a}\right)$ and $V_{\text {eff }}\left(\phi^{a}\right)$. The results are shown in Figs. 16 and 17. Unfortunately, approximating these results via the saddle-point method is not possible due to the lack of a proper expansion point.

We again obtain a Taylor expansion of the classical potential $V_{0}\left(\phi^{a}\right)=-\ln P\left(\phi^{a}\right)$. Expanding (36) around $\phi^{a}=\pi$ to $\mathcal{O}(4)$ yields

$$
\begin{aligned}
V_{0}\left(\phi^{a}\right) \approx & -\ln \left(\frac{2}{\pi}\right)+a(T) \\
& +\left(1+c(T)-\frac{c(T)}{3}-\frac{b^{2}(T)}{2}\right)\left(\frac{\phi^{a}-\pi}{2}\right)^{2} \\
& +\left(\frac{1}{6}+\frac{b^{2}(T)}{6}+\frac{b^{4}(T)}{12}\right)\left(\frac{\phi^{a}-\pi}{2}\right)^{4}
\end{aligned}
$$

In Fig. 18 a comparison of (40) and the simulation for $N_{t}=6$ is shown. A fourth order expansion reproduces the data with high accuracy. An expansion around $\phi=0$ 

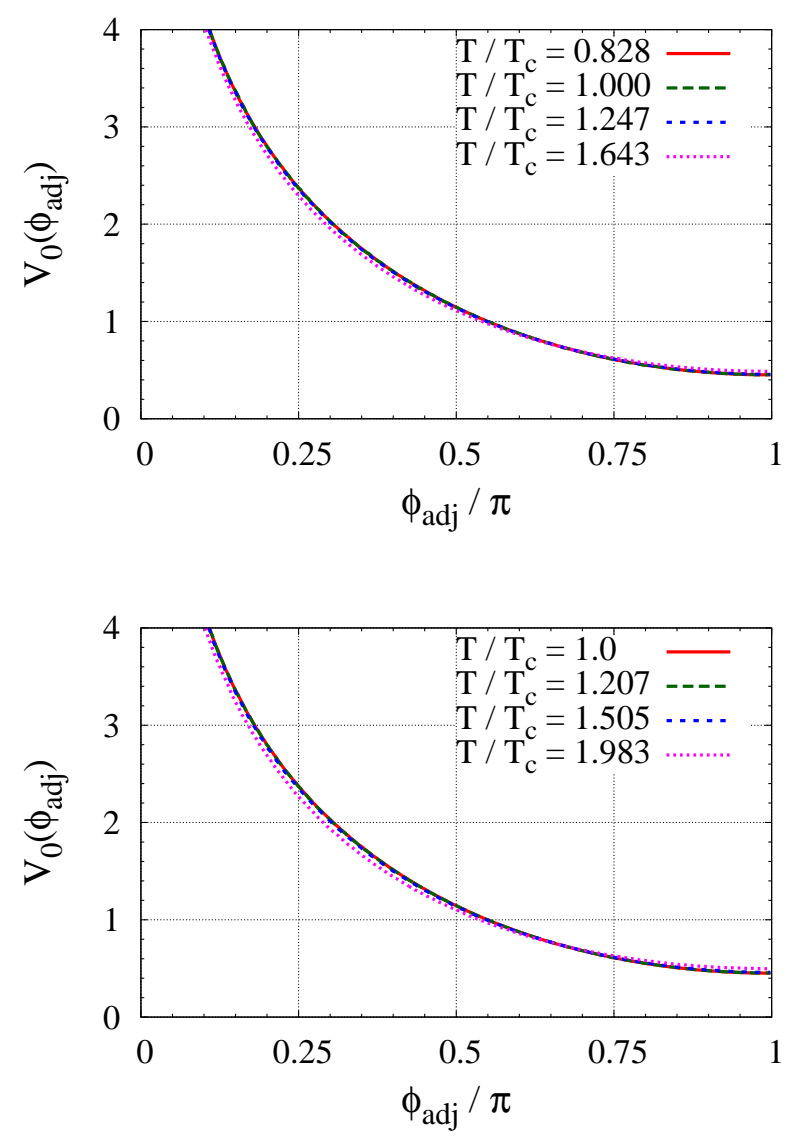

FIG. 15: Constraint effective potential of phase of SU(2) Wilson loop eigenvalues in adjoint representation at $\beta=2.577856$ (top) and $\beta=2.635365$ (bottom). The different temperatures correspond to $N_{t}=12,10,8,6$ respectively.

is not possible due to the logarithmic term in the potential.

\section{SUMMARY AND OUTLOOK}

In this work we simulated pure $\mathrm{SU}(2)$ gauge theory at finite temperature, for two different fixed values of $\beta$ and hence of the lattice spacing. We changed the temperature by changing the time-like extent $N_{t}$ of the lattice. For each temperature we computed the classical potential of the bare Polyakov loop and of the phase of the eigenvalues of the thermal Wilson loop (wrapped around the periodic boundary of the time direction) in the fundamental and adjoint representation, using a histogram method. From these potentials, we obtained the effective potential via Legendre transformation. We obtained model functions for the classical potentials for each case (three parameters are required) and investigated how the parameters depend on temperature and lattice spacing. We discussed how the models can be approximated by
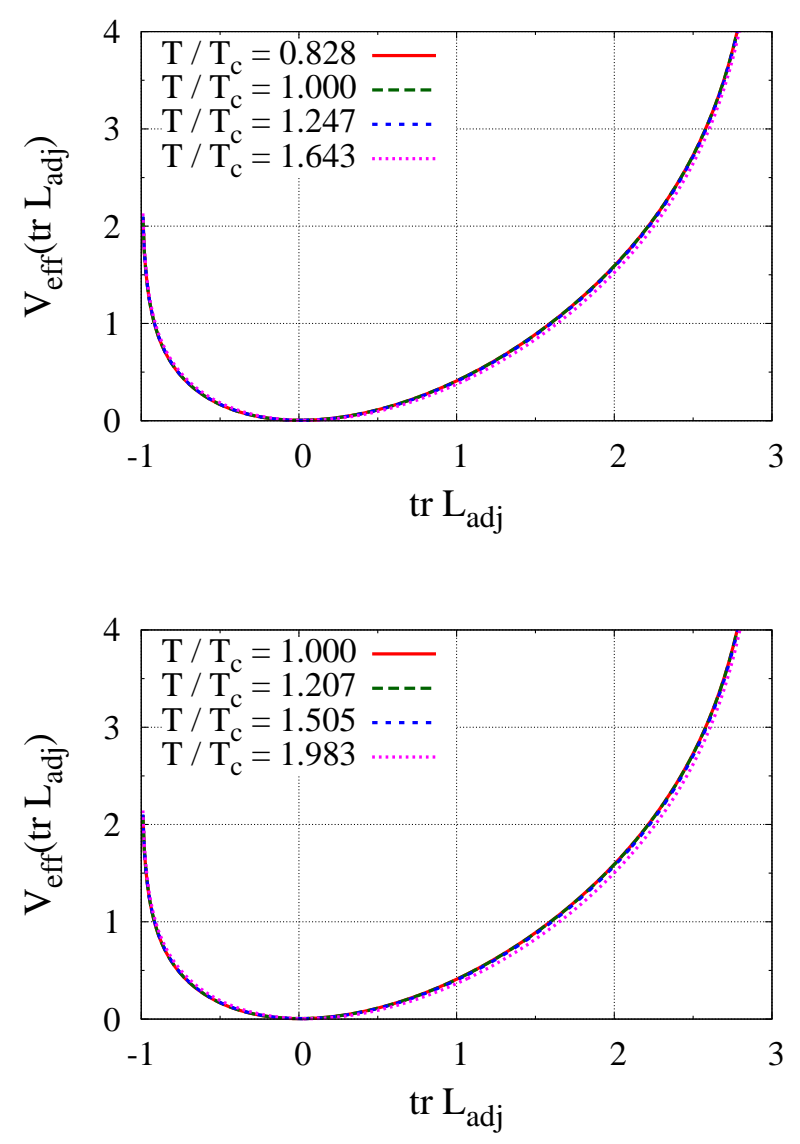

FIG. 16: Effective potential of SU(2) Polyakov loop in adjoint representation at $\beta=2.577856$ (top) and $\beta=2.635365$ (bottom). The different temperatures correspond to $N_{t}=$ $12,10,8,6$ respectively.

Taylor expansions and how they can be converted into one another. We showed how the effective potential of the fundamental loop can be approximated to high precision by analytical expressions, when a next-to-leading order saddle expansion is used to compute the Legendre transformation.

Several conclusions may be drawn from our work: The most obvious conclusion is that both the classical and effective potentials of the bare loop and its eigenvalues are well described by simple models, reminiscent of Landau-Ginzburg theories but with a symmetry breaking term, where the whole dependence on temperature and lattice-spacing is absorbed into three parameters. We have thus confirmed that the ansatz used in Refs. [20, 21] to parametrize the classical potential in a $\mathrm{SU}(2)$ matrix model of Wilson lines is valid also in the full gauge theory, with the same qualitative behavior of the parameters. Also, we have shown that a next-to-leading order approximation of the effective potential of the fundamental loop is reasonable.

We stress that what we have computed in this work is 

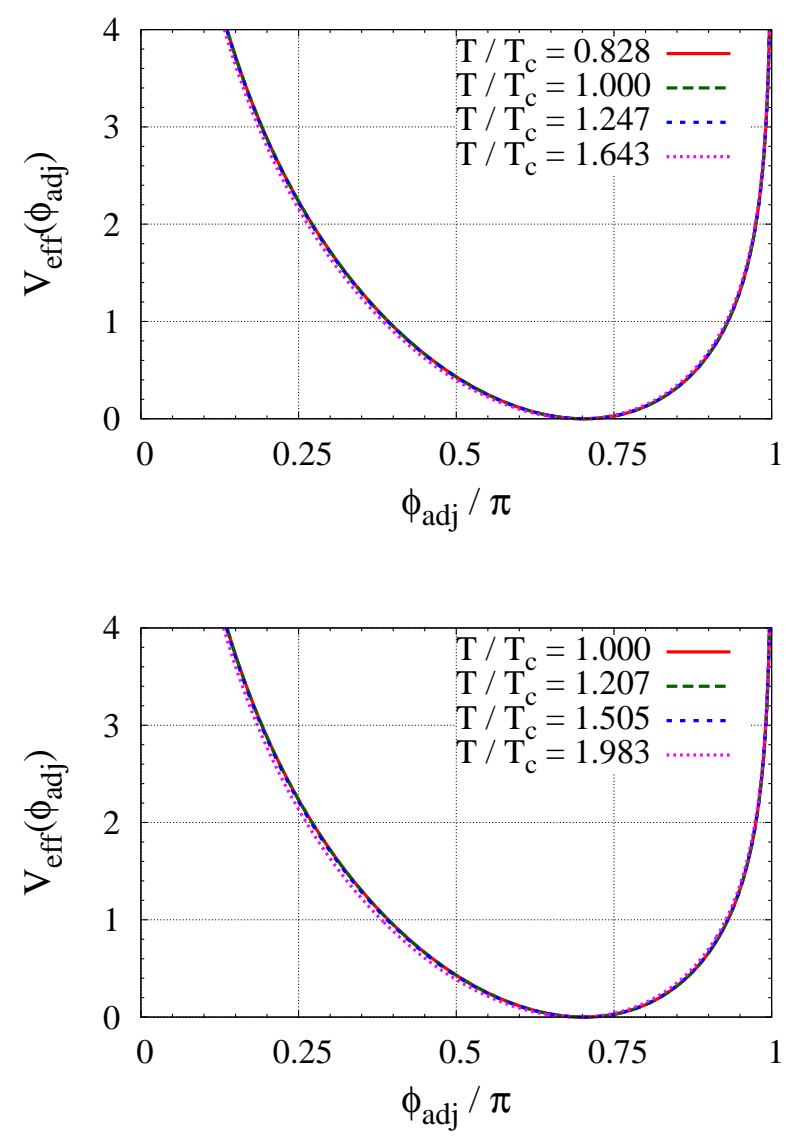

FIG. 17: Effective potential of phase of SU(2) Wilson loop eigenvalues in adjoint representation at $\beta=2.577856$ (top) and $\beta=2.635365$ (bottom). The different temperatures correspond to $N_{t}=12,10,8,6$ respectively.

the effective potential for the bare loop, which vanishes in the continuum limit. In a given irreducible representation of the gauge group, a renormalized loop is extracted from the bare loop by dividing by a renormalization constant, which depends upon the representation [23].

One of the motivations of this work was the idea that the eigenvalues of the Wilson line might be insensitive to renormalization. Our results contradict this. Instead, the eigenvalues of the bare loop are dominated by the effects of the Vandermonde determinant. On the lattice, the Vandermonde determinant arises naturally at each point in spacetime. In the action, its contribution is proportional to $1 / a^{4}$, where $a$ is the lattice spacing, and so it diverges in the continuum limit. In retrospect, it is natural that the lattice effective potential for a site potential is dominated by a Vandermonde term.

For the future, it will be interesting to investigate how the effective potential of renormalized loops can be obtained from our results. Also, one should extract the non-perturbative contribution to the effective potential from our data and compare it to model calculations. Fur-
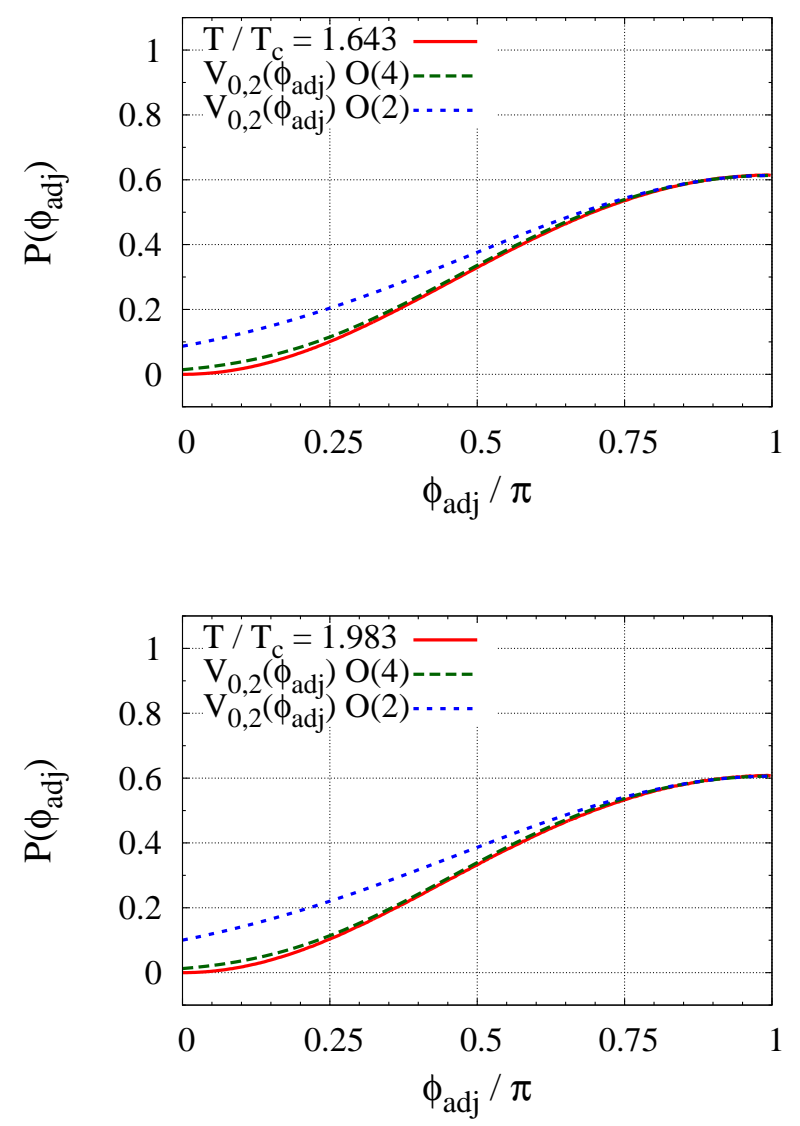

FIG. 18: Distribution of phase of $\mathrm{SU}(2)$ Wilson loop eigenvalues in adjoint representation at $\beta=2.577856$ (top) and $\beta=2.635365$ (bottom). $N_{t}=6$ results compared to Taylor expansions.

thermore, our investigation should be extended to higher representations of $\mathrm{SU}(2)$ and also to other groups, such as $\mathrm{SU}(3)$ or exceptional groups with a trivial center, such as $\mathrm{G}(2)$. Investigating the effects of dynamical Fermions on the results discussed here is currently under way, using standard staggered fermions. It would be interesting to extend this investigation to non-zero chemical potential, which is possible for two colors, where there is no sign problem.

\section{Acknowledgements}

We thank David Scheffler for helpful discussions, for proof-reading and for a script to create histograms.

This work was supported by the Deutsche Forschungsgemeinschaft within SFB 634, by the Helmholtz International Center for FAIR within the LOEWE initiative of the State of Hesse, by the U.S. Department of Energy under contracts \#DE-FG02-09ER41620 and \#DE-AC0298CH10886, by The City University of New York through 
the PSC-CUNY Research Award Program, grant 6651400 44, and by the European Commission, FP7-PEOPLE2009-RG, No. 249203.

All results presented here were obtained using Nvidia GeForce GTX 580 graphics cards.

\section{Appendix A: Determination of physical units}

To fix the physical units for the results discussed in this work we use a standard method which determines the lattice spacing $a$ through measurements of the string tension in lattice units at zero temperature (which in practice means lattices of large volume which are either hypercubic or have $N_{t} \gg N_{x}$ ). In this appendix we give a short summary of the method (which is derived in detail in several textbooks) and present numerical results for $\beta(a)$ and related quantities. Of course the running of the coupling with the cut-off scale has been investigated both analytically and numerically for both twoand three-color gauge theory, in many previous works (see e.g. 32 37 and references therein). Our main motivation for repeating such an investigation is to make use of the advanced computing power of the GPUs to measure at larger $N_{x}$ and $N_{t}$ and with larger statistics as was previously possible and thus reduce systematic and statistical errors. All physical units quoted in this work implicitly refer to the results presented in this appendix.

In pure gauge theory the static quark-antiquark potential is known [38] to be well described by

$$
V(r)=A+\frac{-\pi}{12}(1 / r)+\sigma r+\mathcal{O}\left(1 / r^{2}\right)
$$

The linear coefficient $\sigma$ is the string tension. On the lattice we can measure the potential in lattice units, i.e. $a V\left(a n_{x}\right)$, through the use of rectangular Wilson loops $W\left(n_{x}, n_{t}\right)$ in the $x-t$ plane (our definition of $W$ implies that the trace is taken). These are related to the potential by

$$
\left\langle W\left(n_{x}, n_{t}\right)\right\rangle=e^{-n_{t} a V\left(a n_{x}\right)}\left(1+\mathcal{O}\left(e^{-n_{t} a \Delta E}\right)\right),
$$

where $\Delta E$ is the energy gap between the ground state and the first excited state of a quark-antiquark pair. Since the second term is exponentially suppressed with $n_{t}$, in the limit of large $n_{t}$ the potential can be directly obtained through the use of Creutz ratios

$$
\ln \frac{\left\langle W\left(n_{x}, n_{t}\right)\right\rangle}{\left\langle W\left(n_{x}, n_{t}+1\right)\right\rangle} \approx a V\left(a n_{x}\right) .
$$

Since Eq. A1 is written in lattice units as

$$
a V\left(a n_{x}\right)=a A+\frac{-\pi}{12}\left(1 / n_{x}\right)+\sigma a^{2} n_{x}+\mathcal{O}\left(1 /\left(a n_{x}\right)^{2}\right)
$$

it is clear that what we actually obtain from a $\chi^{2}$ fit of the data is the dimensionless product $a^{2} \sigma \equiv y$. Fixing the string tension $\sigma$ to its physical $T=0$ value (or some other definite value $\sigma_{0}$ ) then determines $a$ for a given $\beta$. The lattice spacing $a$ depends on the choice of $\sigma$ of course, and for theories where this is not directly related to observable physics (as in $\mathrm{SU}(2)$ gauge theory) one in fact often leaves $\sigma$ undefined, which implies that $a$ and all derived quantities are expressed in units of an external parameter.

The crucial point here is that the $\beta$ dependence of the lattice spacing is in fact temperature independent. This implies that once $a(\beta)$ is known at $T=0$, this fixes the temperature (through $T=1 / a N_{t}$ ) and the physical units of all measured quantities for any given $N_{t}$ and $\beta$.

To improve the signal-to-noise ratio for our string tension measurements we employ a method known as APE smearing 39]. This method consists of repeatedly replacing all gauge links $U_{\mu}(n)$ with averages of the form

$$
\tilde{U}_{\mu}(n)=(1-\alpha) U_{\mu}(n)+\frac{\alpha}{6} \sum_{\mu \neq \nu} C_{\mu \nu}(n),
$$

where $C_{\mu \nu}(n)$ represents the staple matrix

$$
\begin{aligned}
C_{\mu \nu}(n) & =U_{\nu}(n) U_{\mu}(n+\hat{\nu}) U_{\nu}^{\dagger}(n+\hat{\mu}) \\
& +U_{\nu}^{\dagger}(n-\hat{\nu}) U_{\mu}(n-\hat{\nu}) U_{\nu}(n-\hat{\nu}+\hat{\mu}),(\mathrm{A}
\end{aligned}
$$

and re-unitarizing the link (projecting back to $S U(N)$ ) after each iteration. The crucial effect of this procedure is to enhance the ground state overlap. APE smearing is applied to a copy of the lattice (the Monte-Carlo thus remains unaffected) on which the Wilson loops are subsequently measured. A good choice for the parameter $\alpha$ is known [40 42] to be

$$
\alpha=1-\frac{1}{1+6 w} ; w=0.2 \rightarrow \alpha=0.5454 \ldots .
$$

We adopt this choice as well and apply the smearing 25 times before measuring Wilson loops on a given lattice configuration.

\section{Results}

We measure $a^{2} \sigma$ on lattices of sizes $24^{3} \times 32,32^{3} \times 48$ and $48^{3} \times 64$, for $\beta$ values ranging from 2.28 to 2.64 using (A3) and (A4). Sample sizes range from measurements on each site of several hundreds (for the larger $\beta$ values) to several tens of thousands (for the smaller $\beta$ values) of independent (smeared) gauge field configurations. From these results we obtain $a \sqrt{\sigma}$, which is shown in Fig. 19, Wilson loops are measured up to spatial and timelike extents of $N_{x} / 2$ and $N_{t} / 2$ respectively. We find consistency with the data sets quoted in Ref. [43].

One must consider potential sources of systematic errors. Firstly there are finite volume and discretization errors, for which we check by comparing the results obtained from lattices of different dimensions. We find that our different choices give consistent results in $\beta$ ranges 


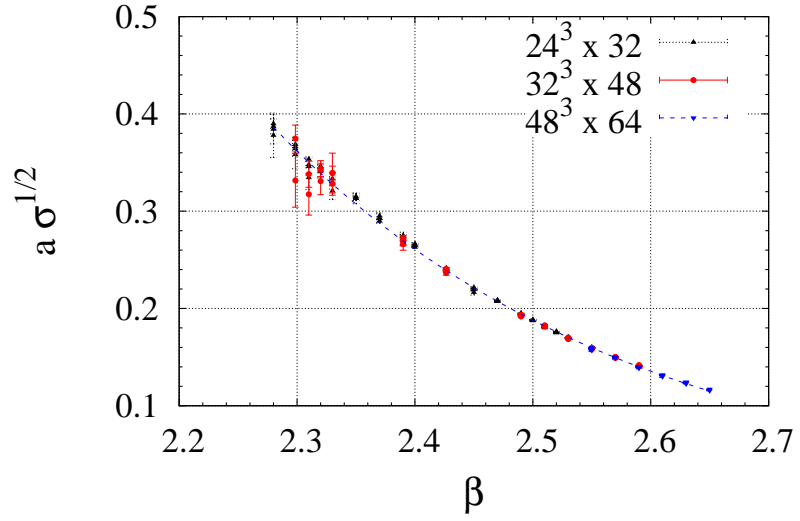

FIG. 19: The running of the lattice spacing with the gauge coupling, computed from $n_{t}=9,10,11,12$ Creutz ratios on different lattices. The line represents $1 / R(\beta)$ (Eqs. (A9|A8)).

where they overlap (at least two different lattices overlap in any given region), therefore we consider these errors to be under control. Second, one must take care to choose the timelike extent $n_{t}$ of the Wilson loops large enough, such that the contribution from the exited states to the Creutz ratios (A3) can be neglected. This is tricky since the signal diminishes with rising $n_{t}$. We find that we can obtain, with reasonably small statistical errors, consistency between $n_{t}=9$ and $n_{t}=10$ on the two smaller lattices for each value of $\beta$. On the largest lattice consistent results are obtained on $n_{t}=11,12$. Fig. 19 plots both respective choices for each lattice as the same dataset. We consider this source of error to be under control as well. The last, and potentially most severe, source of systematic errors are the higher order contributions to the potential (A1), which one must account for. We find that an excellent fit of the data can be obtained by discarding higher order terms and restricting the fit to $n_{x} \geq 2$. Alternatively one could introduce an additional term $\sim B / r^{2}$, or throw away more of the short-distance results. Although we find that the resulting string-tension measurements are much noisier if either of the latter two options is chosen, we find evidence that using $n_{x} \geq 2$ introduces a systematic error such that the string tension is over-estimated (and thus the temperature is under-estimated) for the region $\beta \lesssim 2.45$ if higher-order terms are excluded. We choose to combine two sets of string tension data as our final results. One with $n_{x} \geq 2$ where the next term of (A1) is included and one without such a term but fitting only to $n_{x} \geq 3$. We find that they are consistent. We take this as evidence that the systematic errors from higher-order terms are small for our final results. The data points shown in the figures in this section represent the measurements with the smallest errorbars. To avoid cluttering of the figures, we refrain from showing additional points which were obtained but come with huge errorbars. All points were used however to determine the parameters of the model functions discussed in the following.

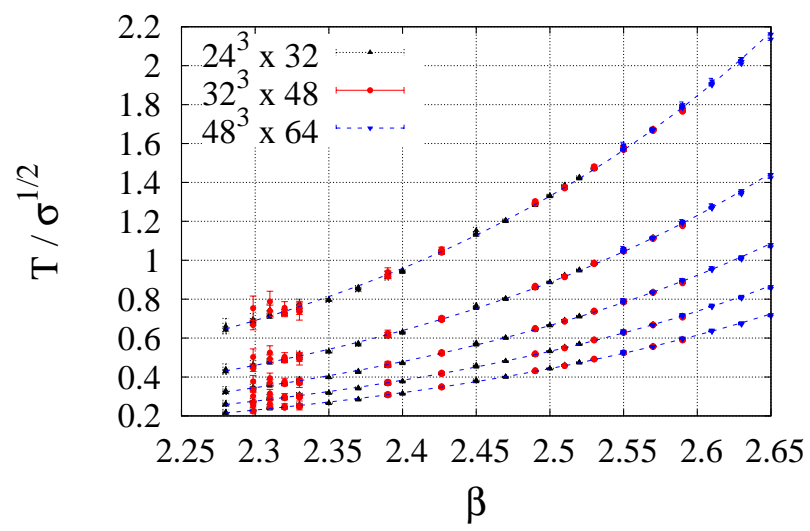

FIG. 20: The physical temperature for $N_{t}=4,6,8,10,12$ (top to bottom). The lines represent $R(\beta) / N_{t}$ (Eqs. (A9|A8)).

Our main goal is to obtain the $\beta$ dependence of $T / T_{c}$ and its inverse for different $N_{t}$. This can be achieved if both $T(\beta)$ for a given $N_{t}$ and $\beta_{c}\left(N_{t}\right)$ are known. Furthermore, it is desirable to obtain not only a finite set of values but model-functions for each of these, valid over a large as possible range. A reasonable approach is to attempt to fit the data with known perturbative formulae, possibly with additional correction terms.

From $a \sqrt{\sigma}$ we directly obtain $T / \sqrt{\sigma}=1 /\left(a \sqrt{\sigma} N_{t}\right)$. The results are plotted in Fig. 20 for different $N_{t}$. We find that it is possible to model our results by taking the inverse of the leading logarithmic term of the perturbative running-coupling formula (see e.g. [33]). Defining

$$
R(\beta)=\exp \left(\frac{\beta-d}{b}\right)
$$

and using

$$
a \sqrt{\sigma}=\frac{1}{R(\beta)}, \quad \frac{T}{\sqrt{\sigma}} N_{t}=R(\beta) .
$$

we find that our data shown in Figs. 19 and 20 are well described for the entire range of $\beta$ considered with the parameters

$$
d=1.98(1) \quad, \quad b=0.305(6) \text {. }
$$

This is similar to what was done in Ref. 44 although the $\beta$ range considered here is larger. It is in fact somewhat amazing that a leading-order formula works so well over such a large range. It is also very convenient, since Eq. (A8) can be easily inverted. If we restrict our fit-window to the same as was used in [44], we obtain a consistent value for the parameter $b$. If we know a $\beta_{C}$ for a given $N_{t}$ we are done, for we can then obtain

$$
\frac{T}{T_{c}}=\frac{R(\beta)}{R\left(\beta_{c}\right)}=\exp \left(\frac{\beta-\beta_{c}}{b}\right),
$$


and likewise

$$
\log \left(\frac{T}{T_{c}}\right) b+\beta_{c}=\beta .
$$

Our numerical results for $T / \sqrt{\sigma}$ can analogously be converted to $T / T_{c}$. The parameter $\sigma$ drops out entirely.

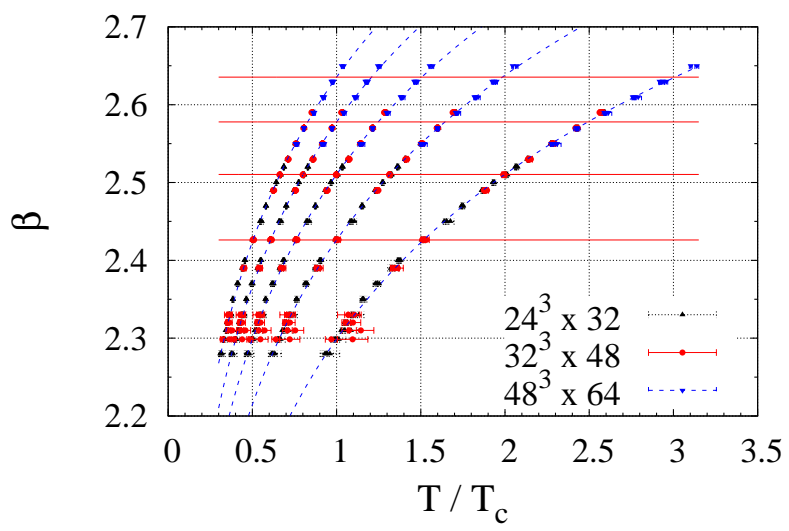

FIG. 21: The running coupling for $N_{t}=12,10,8,6,4$ (left to right). The horizontal lines mark $\beta_{C}$ for $N_{t}=12,10,8,6$ (top to bottom). The model function is defined in Eq. A12.

We show $\beta$ as a function of $T / T_{c}$ in Fig. 21, both as raw data and model-function. We collected $\beta_{c}$ values for $N_{t}=4,5,6,8,12,16$ from Refs. [43, 45, 46] and fit them to interpolate for other $N_{t}$ values, using

$$
\beta_{c}\left(N_{t}\right)=a_{0}+b_{0} \log \left(N_{t}\right)-\log \left(\log \left(N_{t}\right)\right)^{c_{0}},
$$

We find that the next-to-leading logarithm is necessary to appropriately describe such a range of $N_{t}$. Our fitparameters converge to

$$
a_{0}=1.1579(6), b_{0}=0.9398(1), c_{0}=1.627(2) \text {, }
$$

with $\chi^{2} /$ dofs $\approx 0.18$. Eq. A13 is used in Eq. A11 to determine $\beta_{c}$ for all $N_{t}$ instead of the raw data.

One last thing should be mentioned: We realize that the results presented in this appendix go beyond what is necessary for the study presented in the main sections. In particular, to obtain $T / T_{c}$, when simulating on a lattice of time-like extent $N_{t}$ at a given $\beta$ which is known to be $\beta_{c}$ for a different $N_{t}^{c}$, one can simply use the relation

$$
\frac{T}{T_{c}}=\frac{N_{t}^{c}}{N_{t}} .
$$

We conducted the investigation discussed here hoping that it will be useful in a more general setting and we have chosen to present $T / T_{c}$ values throughout this paper as our general method provides them. Equation A15 however is very useful to us to estimate errors. It turns out that the $T / T_{c}$ values quoted throughout this paper differ by no more than $\sim 2 \%$ from what A15 predicts, which is quite satisfactory.
[1] G. 't Hooft, Nucl. Phys. B 138, 1 (1978).

[2] A. M. Polyakov, Phys. Lett. B 72, 477 (1978).

[3] L. Susskind, Phys. Rev. D 20, 2610 (1979).

[4] L. D. McLerran and B. Svetitsky, Phys. Rev. D 24, 450 (1981).

[5] A. Dumitru, J. Lenaghan and R. D. Pisarski, Phys. Rev. D 71, 074004 (2005) hep-ph/0410294.

[6] A. Dumitru, Y. Hatta, J. Lenaghan, K. Orginos and R. D. Pisarski, Phys. Rev. D 70, 034511 (2004) hep-th/0311223.

[7] P. N. Meisinger, T. R. Miller and M. C. Ogilvie, Phys. Rev. D 65, 034009 (2002) hep-ph/0108009.

[8] P. N. Meisinger and M. C. Ogilvie, Phys. Rev. D 65, 056013 (2002) hep-ph/0108026.

[9] R. D. Pisarski, Phys. Rev. D 74, 121703 (2006) hep-ph/0608242.

[10] A. Dumitru, Y. Guo, Y. Hidaka, C. P. K. Altes and R. D. Pisarski, Phys. Rev. D 83, 034022 (2011) arXiv:1011.3820 [hep-ph]].

[11] A. Dumitru, Y. Guo, Y. Hidaka, C. P. K. Altes and R. D. Pisarski, Phys. Rev. D 86, 105017 (2012) arXiv:1205.0137 [hep-ph]].

[12] T. Umeda, S. Ejiri, S. Aoki, T. Hatsuda, K. Kanaya, Y. Maezawa and H. Ohno, Phys. Rev. D 79, 051501 (2009) arXiv:0809.2842 [hep-lat]].

[13] M. Panero, Phys. Rev. Lett. 103, 232001 (2009)
arXiv:0907.3719 [hep-lat]].

[14] S. Datta and S. Gupta, Phys. Rev. D 82, 114505 (2010) arXiv:1006.0938 [hep-lat]].

[15] S. Borsanyi, G. Endrodi, Z. Fodor, S. D. Katz and K. K. Szabo, JHEP 1207, 056 (2012) arXiv:1204.6184 [hep-lat]].

[16] M. Caselle, L. Castagnini, A. Feo, F. Gliozzi, U. Gursoy, M. Panero and A. Schafer, JHEP 1205, 135 (2012) arXiv:1111.0580 [hep-th]].

[17] B. Lucini and M. Panero, Phys. Rept. 526, 93 (2013) arXiv:1210.4997 [hep-th]].

[18] L. Fister and J. M. Pawlowski, arXiv:1301.4163 [hep-ph].

[19] J. Braun, H. Gies and J. M. Pawlowski, Phys. Lett. B 684, 262 (2010) arXiv:0708.2413 [hep-th]].

[20] D. Smith, Phys. Rev. D 82, $034503 \quad$ (2010) arXiv:0911.4037 [hep-lat]].

[21] D. Smith (2010), Lattice simulation of a center symmetric three dimensional effective theory for SU(2) YangMills, Ph.D. thesis, Goethe University of Frankfurt, Germany.

[22] A. D. Kennedy and B. J. Pendleton, Phys. Lett. B 156, 393 (1985).

[23] S. Gupta, K. Huebner and O. Kaczmarek, Phys. Rev. D 77, 034503 (2008) arXiv:0711.2251 [hep-lat]].

[24] C. S. Fischer, L. Fister, J. Luecker and J. M. Pawlowski, arXiv:1306.6022 [hep-ph]. 
[25] K. Langfeld and J. M. Pawlowski, arXiv:1307.0455 [heplat].

[26] D. Diakonov, C. Gattringer and H. -P. Schadler, JHEP 1208, 128 (2012) arXiv:1205.4768 [hep-lat]].

[27] B. A. Berg, Markov Chain Monte Carlo Simulations and Their Statistical Analysis, World Scientific Publishing, Singapore, 2004

[28] J. Greensite, Phys. Rev. D 86, 114507 (2012) arXiv:1209.5697 [hep-lat]].

[29] J. Greensite and K. Langfeld, arXiv:1301.4977 [hep-lat].

[30] A. Dumitru and D. Smith, Phys. Rev. D 77, 094022 (2008) arXiv:0711.0868 [hep-lat]].

[31] D. Smith, Nucl. Phys. A 820, 227 (2009) arXiv:0810.1129 [hep-lat]].

[32] G. S. Bali and K. Schilling, Phys. Rev. D 47, 661 (1993) hep-lat/9208028.

[33] J. C. R. Bloch, A. Cucchieri, K. Langfeld and T. Mendes, Nucl. Phys. B 687, 76 (2004) hep-lat/0312036.

[34] C. Michael, Phys. Lett. B 283, 103 (1992) hep-lat/9205010.

[35] A. Cucchieri, hep-lat/0209076

[36] M. Luscher, R. Sommer, P. Weisz and U. Wolff, Nucl.
Phys. B 413, 481 (1994) hep-lat/9309005.

[37] S. Necco and R. Sommer, Phys. Lett. B 523, 135 (2001) hep-ph/0109093.

[38] M. Luscher and P. Weisz, JHEP 0207, 049 (2002) hep-lat/0207003.

[39] M. Albanese et al. [APE Collaboration], Phys. Lett. B 192, 163 (1987).

[40] N. Cardoso and P. Bicudo, J. Comput. Phys. 230, 3998 (2011) arXiv:1010.4834 [hep-lat]].

[41] F. Geles and C. B. Lang, arXiv:1103.5368 [hep-lat].

[42] C. Morningstar and M. J. Peardon, Phys. Rev. D 69, 054501 (2004) hep-lat/0311018.

[43] J. Fingberg, U. M. Heller and F. Karsch, Nucl. Phys. B 392, 493 (1993) hep-lat/9208012.

[44] P. de Forcrand and L. von Smekal, Phys. Rev. D 66, 011504 (2002) hep-lat/0107018.

[45] A. Velytsky, Int. J. Mod. Phys. C 19, 1079 (2008) arXiv:0711.0748 [hep-lat]].

[46] X. Cheng and E. T. Tomboulis, Phys. Rev. D 86, 074507 (2012) arXiv:1206.3816 [hep-lat]]. 